\title{
microFE-BCl: An Asynchronous Practical EEG-based Control Paradigm Assisted by Micro-facial-expressions
}

This paper was downloaded from TechRxiv (https://www.techrxiv.org).

\section{LICENSE}

CC BY 4.0

SUBMISSION DATE / POSTED DATE

$10-04-2021 / 16-04-2021$

\section{CITATION}

Lu, Zhufeng (2021): microFE-BCI: An Asynchronous Practical EEG-based Control Paradigm Assisted by Micro-facial-expressions. TechRxiv. Preprint. https://doi.org/10.36227/techrxiv.14397593.v1

$\mathrm{DOI}$ 


\title{
microFE-BCl: An Asynchronous Practical EEG-based Control Paradigm Assisted by Micro-facial-expressions
}

\author{
Xiaodong Zhang, Member, IEEE, Zhufeng Lu, Hanzhe Li, Teng Zhang, Linxia Gu, and Yachun \\ Wang
}

\begin{abstract}
In this study, an EEG-based control paradigm assisted by micro-facial-expressions (microFE-BCl) was developed, focusing on the mainstream defect as the insufficiency of real-time capability, asynchronous logic, and robustness. The core algorithm in microFE-BCl contained two stages (asynchronous 'ON' detection \& microFE-BCl based real-time control) with four steps (obvious non-microFE-EEGs exclusion, interface 'ON' detection, microFE-EEGs real-time decoding, and validity judgment). It provided the asynchrounous function, decoded 8 instructions from the latest $100 \mathrm{~ms} E E G s$, and greatly reduced the frequent misoperation. In the offline assessment, microFE-BCl achieved $96.46 \% \pm 1.07$ accuracy for interface 'ON' detection and $92.68 \% \pm 1.21$ for microFE-EEGs real-time decoding, with the theoretical output timespan less than $200 \mathrm{~ms}$. This microFE-BCl was implemented into a software, and applied to two online manipulations for evaluating the stability and agility. In object-moving with a robotic arm, the averaged loU was $60.03 \pm 11.53 \%$. In water-pouring with a prosthetic Hand, the averaged water volume was $202.5 \pm 7.0 \mathrm{ml}$. During online, microFE-BCl performed no significant difference $(P=0.6521 \& P=0.7931)$ with commercial control methods (i.e., FlexPendant and Joystick), indicating a similar level of controllability and agility. This study demonstrated the capability of microFE-BCl, enabling a novel solution to the noninvasive $\mathrm{BCls}$ in real-world challenges.
\end{abstract}

Index Terms - asynchronous, BCl, EEG, EEG-based control, microexpression

\section{INTRODUCTION}

$\mathrm{E}$ LECTROENCEPHALOGRAM (EEG), ever since it was $\checkmark$ first proposed by Berger $\cdot H$ in 1929 [1], has been studied for nearly a hundred years. The EEG's unique character of representing human intention spontaneously and unconsciously has been used to regulate human-machine interaction in a natural way. Much research efforts has poured into operating peripherals with EEGs, namely EEG-based Control, also well-known as a typical noninvasive branch of brain-computer interface (BCI) [2]. Non-invasive EEGs are considered to have lower cost and higher universal application potentials [3]. Several paradigms have been developed to arouse typical responses of the brain activity and enhance the salient features under certain events or stimulus [4]. The basic form of EEG-based Control could be brought into reality at the current level of technology. Encouraged by the significant potential of EEG-based Control [5], research areas related to paradigm development and EEG interaction have attracted increasing attentions

To realize the EEG-based Control with a low signal-tonoise ratio (SNR) non-invasive surface EEGs, several mainstream paradigms have been developed, such as Steady State Visual Evoked Potential (SSVEP), Motor Imagery (MI) and so on [6]. SSVEP-BCIs realize the conversion from human intentions into machine instructions by decoding the

- X. Zhang, Z. Lu, H. Li, T. Zhang, and Y. Wang are with Shaanxi Key Laboratory of Intelligent Robot, School of Mechanical Engineering, Xi'an Jiaotong University, Xi'an, Shannxi, China. E-mail: zhufeng.luk@qq.com

- L. Gu, is with Department of Biomedical and Chemical Engineering and Sciences, College of Engineering \& Science, Florida Institute of Technology, Florida, America. E-mail: gul@fit.edu oscillatory components in EEGs within the primary visual cortex which match the frequency or harmonics of the repetitive visual stimulus, and establishing the correspondence between command sets and visual stimuli [7]. Early in 2008, Muller-Putz proposed an SSVEP-based 2-DoFs neuro-prosthesis with the ability to perform 4 types of hand movements [8]. In 2020, Han Jin developed an innovative BCI-controlled robot arm for both ideogram and phonogram writing, with an improved hybrid SSVEP paradigm, containing 108 commands [9]. Besides the usage in device control [10-12], SSVEP-BCIs and its variants were typically adopted in screen spellers for its characteristic in receiving visual stimulations [13]. MI-BCIs establishe the correspondence between peripheral actions and imaginary movement by dealing with the event-related (de)synchronization (ERD/ERS) within the motor cortex under specific mental imagination [14]. MI-BCIs are widely used in the motor recovery after stroke and the control of alternative prostheses, considering the natural mapping relationship between MI and the motor cortex [15]. The first MI-based prosthesis including two classes operated by a patient with tetraplegia was reported by Pfurtscheller in 2000 [16]. The decoding of 4 complex right-hand MI tasks was reported by Edelman B J in 2016 including flexion, extension, supination, and pronation, which leaded to the development of naturalistic and intuitive MIs [17]. In addition, hybrid BCIs, as an improvement of unitary paradigm are developed rapidly [18], such as integrated MI and SSVEP [19], intergrated P300 and SSVEP [20], and so on.

The mainstream paradigms, such as SSVEP and MI, face 
challenges to be transformed to peripheral operations with daily practicability. Specifically, SSVEP requires additional visual stimulator and period for steady-state visual evocation. The MI requires extra adaption to paradigm-self and highly concentration. The EEG-based control was considered as a promising control method, besides its primary function as a translator from human intention to machine instructions. Moreover, it needs to satisfy the basic requirements of real-time, precision, user-friendliness, and easiness, similar to the traditional control approaches. An EEG-based control paradigm assisted by facial-expression (FE-BCI) proposed by our research group [21-25] provides the capability for real-time decoding and control (each output generated from the latest $100 \mathrm{~ms}$ EEGs) [21], and has the characteristics of no additional user adaption, no stimulators, and no nerve adaptability [23].

In this work, an EEG-based control paradigm assisted by micro-facial-expressions (microFE-BCI) is proposed to improve its practical performance for comprehensive and complex daily situations. Both offline and online experiments were conducted. The effective connectivity analysis of microFE-EEGs was demonstrated to delineate the interaction among EEGs under microFE-BCI. The methodology of microFE-BCI, including computation logic, asynchronous strategy, and detailed steps, was illustrated. The offline performance and online controllability were assessed. The novel aspects of this new paradigm are: 1) An integral asynchronous BCI strategy is developed to enable users to switch on/off the paradigm in their will; 2) The microexpressions provides the possibility to distinguish the facial expressions in daily communication during EEG-basedcontrol, and also increases the aesthetics, which ensures the suitability for patients who can hardly complete exaggerated facial expressions; 3 ) The instruction sets are expanded with the support of an improved core algorithm, combining the deep-learning framewrok; 4) A validity judgment step is added to decrease the frequent misoperations exposed in the traditional literal translation mode.

\section{Materials}

\subsection{EEG Recording}

The commercial EEG acquisition system (Neuracle Technology Co., Ltd.) has 34 electrodes (including 30 EEG

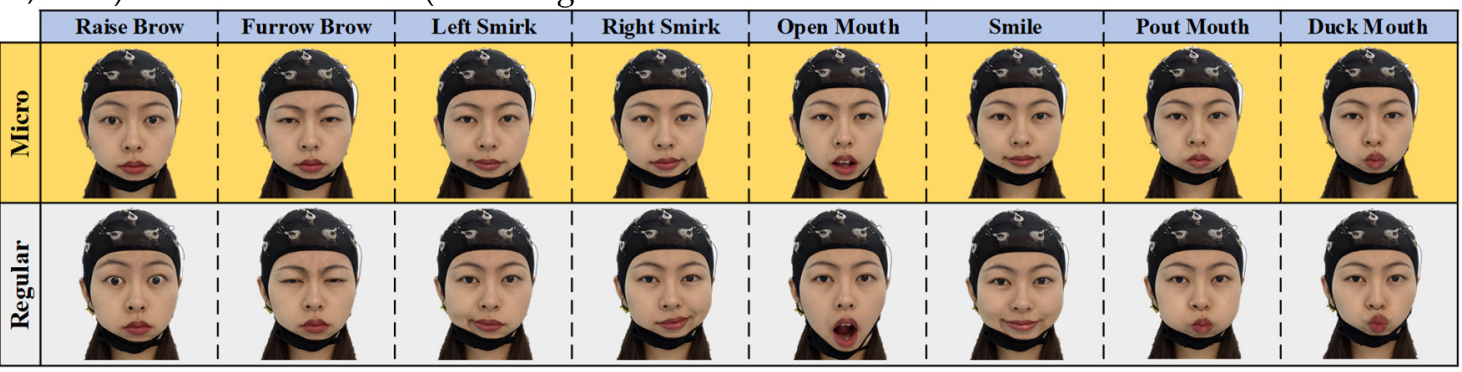

Fig. 2. The 8 microFEs adopted in this research and their comparison with the regular amplitude.

\section{- Section one: Microexpression}

8 microFEs were performed by each subject following the on-screen prompts. Each microFE was conducted for 5 sessions consisted of 8 trials. A total of 40 trials were completed by each subject for one single microFE.

In each trial, $3 \mathrm{~s}$ countdown, $4 \mathrm{~s}$ resting-state, $4 \mathrm{~s}$ channels, 1 reference, 1 ground, and 2 EOG channels, following the international 10-20 electrode location system) with $1000 \mathrm{~Hz}$ sampling rate and $\mathrm{WiFi}(5 \mathrm{G})$ wireless communication (Fig. 1). The reference was placed at $\mathrm{CPz}$, and the ground was placed at AFz [26]. During the experiment, impedances were kept below $5 \mathrm{k} \Omega$.
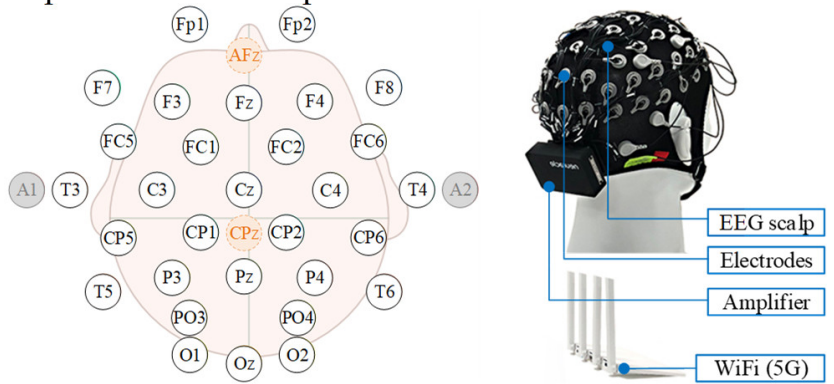

Fig. 1. The Neuracle device and its electrode placement. By system default, $A f z$ is the ground, $C P z$ is the reference, and $A 1, A 2$ are EOGs.

\subsection{Subjects}

Sixteen healthy subjects (25-38 years of age, 13 males and 3 females) participated in the experiments, without any known cognitive deficits and prior experience. Written informed consent was signed ahead, and all experiments were conducted in accordance with the Declaration of Helsinki [27]. These sixteen subjects were divided into two groups, where six subjects (S1-S6) participated only in the offline experiment for offline algorithm assessment and software development, while other ten subjects (S7-S16) participated directly in the online experiment to verify the practicability of the microFE-BCI.

\subsection{Offline Experimental Procedure}

To develop an asynchronous EEG-based control method, EEGs under resting-state, aroused by microexpressions and under relaxing-state were collected. To expand the instruction set, a total of 8 easy-to-execute microexpressions (microFEs) were adopted. In addition, experiments with the same facial expressions but in regular amplitude were also conducted as case comparison. An instruction video was displayed in beginning to illustrate the difference between microexpression and facial expression in regular amplitude. Fig. 2 illustrates the 8 microFEs and their comparison with the regular amplitude.

microexpression-state and $4 \mathrm{~s}$ relaxing-state were contained in turn. The switch among states was accompanied by different beepers and screen prompts. At the restingstate, subjects were asked to avoid extra movements. At the microexpression-state, subjects followed the on-screen prompts to make the designated microFE and hold it for 4 
s. At the relaxing-state, subjects were free for any volunteered movement, including but not limited to talking, drinking, reading, writing, physical activity, and so on. The timing diagram for one trial is demonstrated in Fig. 3.

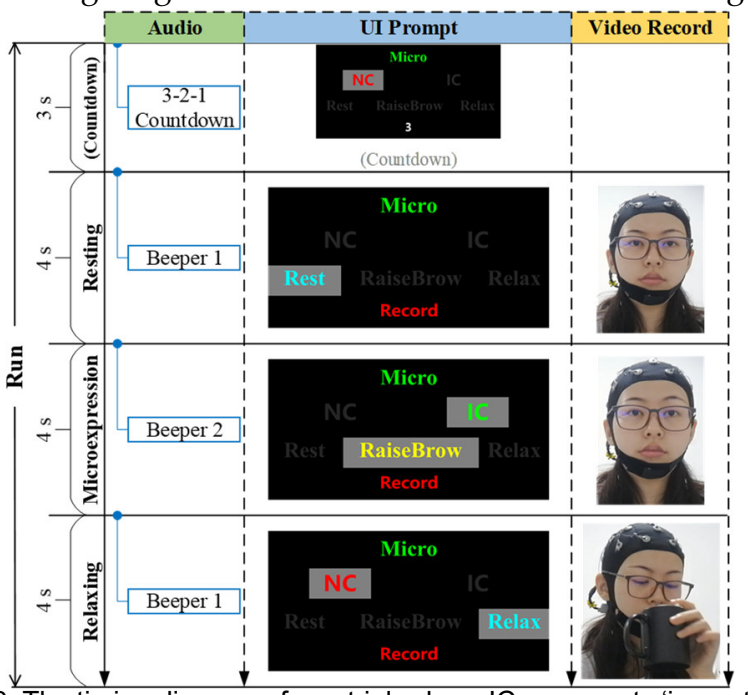

Fig. 3. The timing diagram of one trial, where IC represents 'in-control' and NC represents 'non-control', for details please refer to ALGORITHM.

\section{- Section two: Facial expression (regular amplitude)}

In the regular amplitude section, the same session settings as the microFE phase were adopted. For comparison, each facial expression was conducted for only 2 sessions, in other words a total 16 trials.

- Section three: Supplemental EEGs at Relaxing-state Noticing that some subjects tended not to perform any volunteered movements at the relaxing-state, 3 additional

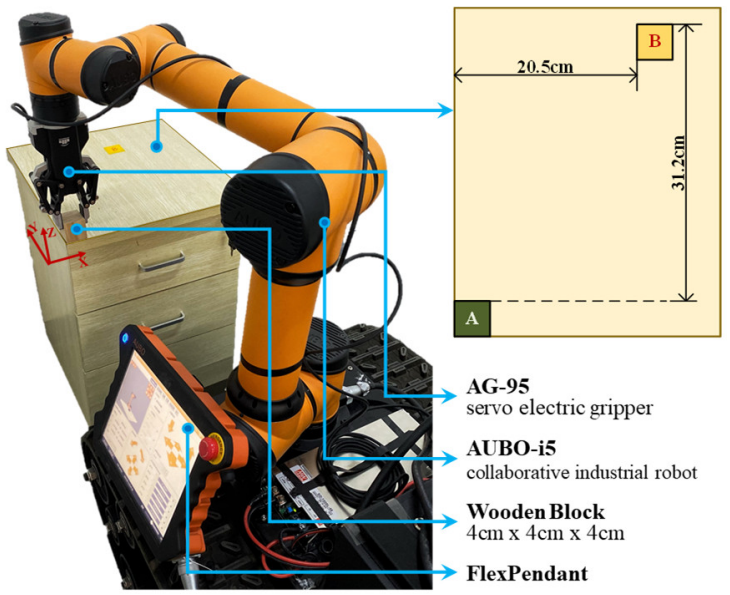

(a) The initial state of the assembled robotic arm system in Task one (object-moving), and the plan view of the position A \& B.

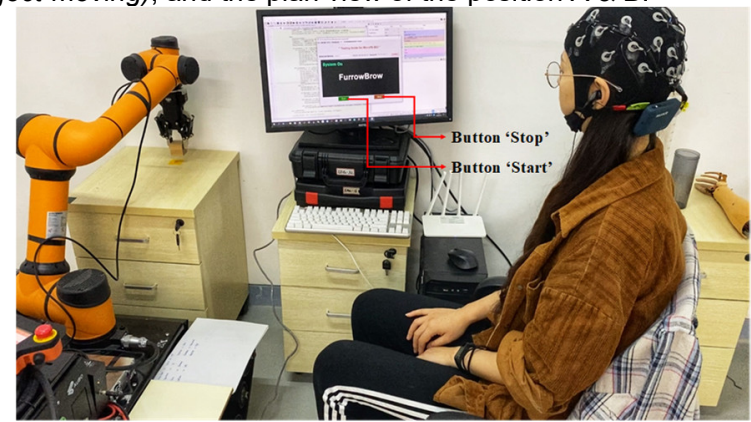

(c) Experimental scene graph for Task one (object-moving).

Fig. 4. The illustration for online experiment. trials of EEGs under relaxing were collected, including 1 trial for reading \& talking, 1 trial for drinking \& eating, and 1 trial for physical moving. Each trial lasted for 96 seconds.

\subsection{Online Experimental Procedure}

After the offline experiment and its assessment, a software for microFE-BCI was developed. To verify the feasibility and the practicality of microFE-BCI for peripheral application, subjects S7-S16 participated in the online experiment directly without any prior experience with the offline.

Two specific external device manipulation tasks, atypical versus daily, focused on stability and agility respectively, were conducted. In both tasks, EEGs were sent wirelessly by WiFi to the computer (Windows 10, i5-6500 CPU, 3.20GHz, GTX 960 GPU). The stepping control were adopted by both devices. Before manipulation, training data for calculating the individual parameters and classifiers were collected first, of which the acquisition process is consistent with the offline experiment but with fewer trials and being more casual.

\section{- Task one: Object-moving with a Robotic Arm}

In this task, subjects were required to use this proposed microFE-BCI to first switch on the system, then operate the robotic arm to move a wooden block $\left(4 \mathrm{~cm}^{*} 4 \mathrm{~cm}^{*} 4 \mathrm{~cm}\right)$ from its initial position A (bottom left corner of the desk) to the position B (as illustrated in Fig. 4(a)), and then switch off the system. The robotic arm used was the assembly of an AUBO-i5 collaborative industrial robot (6 DoFs, provided by AUBO Robotics China Co., Ltd.) equipped with an AG-95 servo-electric gripper (provided by DH-Robotics Technology Co. Ltd), as shown in Fig. 4(a).

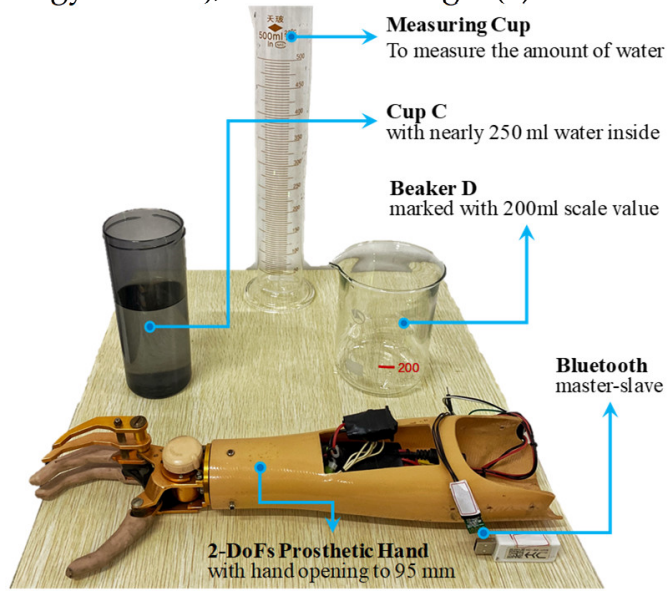

(b) Experiment scene layout and the 2-DoFs prosthetic hand of Task two (water-pouring).

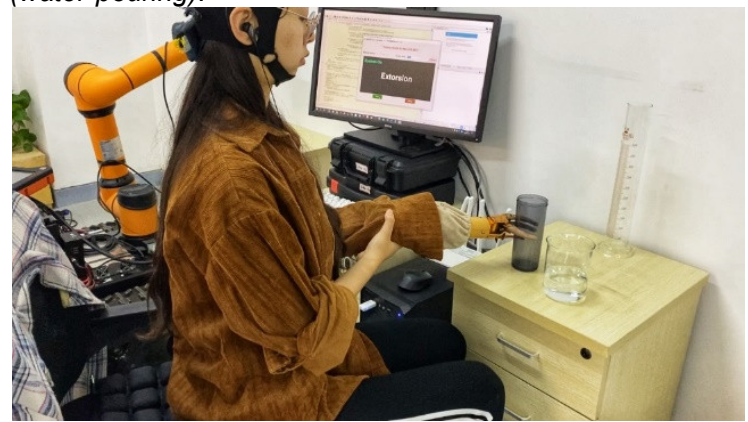

(d) Experimental scene graph for Task two (water-pouring). 
Before each trial, the robotic arm restored its initial state, with the block grabbed (Fig. 4(a)). To move the block, subjects need to first lift it, then adjust its position, and finally lower it and open the electric gripper to ensure a stable placement. For operation details please refer to RESULTS. The experimental scene is graphed in Fig. 4(c).

Before starting, subjects first had 5 mins to get familiar with the operation for microFE-BCI based robotic arm, then this task was repeated for 5 trials. In each trial, subjects began to complete the task after pressing the 'Start' button on the GUI by themselves. If the task was not finished within 5 mins or the block was fell halfway, this trial would be deemed as a failure. The deviation for the same task but using FlexPendant was recorded as a reference.

\section{- Task two: Water-pouring with a Prosthetic Hand}

In this task, subjects were required to use the microFE-BCI to first switch on the system, then operate the prosthetic hand to pour $200 \mathrm{ml}$ water from cup C to beaker D, and then switch off the system. The prosthetic hand used was customized by Danyang Artificial Limb Co., Ltd. with 2 DoFs (wrist and finger joints) and Bluetooth module, as shown in Fig. 4(b).

Before each trial, the prosthetic hand was kept in front of the cup $C$ with the hand opened to the maximum (95 $\mathrm{mm})$, and the water initially stored in the cup C was nearly $250 \mathrm{ml}$, as in Fig. 4(b). To pour the water, subjects need to grasp the cup $C$ first, then lift and whirl it to transfer demanded amount of water, and finally slightly lower it and open the palm. Fig. 4(d) shows the experimental scene.

Similarly, subjects had 5 mins first to get familiar with the, then this task was repeated for 5 trials. Each trial started with the 'Start' button being pressed, and would be viewed as a failure if not be completed within 3 mins or cup was dropped halfway. The deviation to perform water-pouring with this prosthetic hand but controlled by Joystick was also recorded as reference.

\section{BRAIN CONNECTIVITY UNDER MICROFE-EEGs}

Brain connectivity assesses the integration of cerebral areas by identifying variations in activation within and interactions between brain areas [28, 29]. As one major aspect, effective connectivity is defined as the direct or indirect influence that one neural system exerts over another [30]. To obtain a better insight into this microFE-BCI, Orthogonalized Partial Directed Coherence (OPDC) was used to measure the dynamic directional interactions among brain regions under microFEs.

\subsection{Orthogonalized Partial Directed Coherence}

OPDC, as an orthogonalized version of the classical Partial Directed Coherence (PDC) [31], focuses on reducing the spurious co-variability resulted from the similarity in several EEGs affected by volume conduction [32], which may be falsely perceived as connectivity [33]. OPDC developed out of the concept of Granger-causality [34], investigating the information flow within coupled dynamical networks based on multivariate autoregressive (MVAR) models [35], detecting not only direct but also indirect pathways linking interacting brain regions [36]. Based on the dual extended
Kalman filter-based time-varying PDC analysis [37], by orthogonalizing the imaginary part of the coherence [32], OPDC mitigates the common result of volume conduction effects. The estimating process of OPDC is as follows [38]: 1) MVAR model

For time series $y(n) \in \mathbb{R}^{\text {ch }}$ with $L$ samples (where $n=1, \ldots, L)$, the MVAR model is defined as [39]:

$$
\left[\begin{array}{c}
y_{1}(n) \\
\vdots \\
y_{c h}(n)
\end{array}\right]=\sum_{r=1}^{m}\left[\begin{array}{ccc}
a_{1,1}^{r} & \cdots & a_{1, c h}^{r} \\
\vdots & \ddots & \vdots \\
a_{c h, 1}^{r} & \cdots & a_{c h, c h}^{r}
\end{array}\right]\left[\begin{array}{c}
y_{1}(n-r) \\
\vdots \\
y_{c h}(n-r)
\end{array}\right]+\left[\begin{array}{c}
w_{1}(n) \\
\vdots \\
w_{c h}(n)
\end{array}\right]
$$

where $\left[\begin{array}{lll}\mathrm{y}_{1}(n) & \cdots & y_{c h}(n)\end{array}\right]^{\mathrm{T}}$ is the current value of each time series, $c h$ denotes the number of channels, the real-valued parameter $a_{p, q}^{r}(p=1, \ldots, c h ; q=1, \ldots, c h)$ in matrices $A_{r}$ represents the predictor coefficient between the current value of channel $p$ and the past information of channel $q$ at the delay $r, m$ is the model order indicating the number of previous data points used for modeling, and $\left[\begin{array}{lll}w_{1}(n) & \cdots & w_{c h}(n)\end{array}\right]^{\mathrm{T}}=w(n)$ is a normally distributed realvalued zero-mean white noise vector representing onestep prediction error.

The instantaneous effect among channels is explained by correlations among the off-diagonal element within the covariance of $w(n)$ [40]. The optimum order $m$ was estimated using Akaike information criterion (AIC) [41].

\section{2) Time-varying generalized OPDC (gOPDC)}

To detect the coherence between channels in discrete frequency, $A_{r}$ in the time-varying MVAR model is transformed into frequency domain:

$$
A(n, f)=\mathbf{I}-\left.\sum_{r=1}^{m} A_{r}(n) z^{-r}\right|_{z=e^{j 2 \pi f}}
$$

where $\mathbf{I}$ is the identity matrix and the frequency $f$ varies from 0 to the Nyquist rate. To alleviate the effect of mutual sources within surface EEGs affected by spatial smearing in the tissue layer, the orthogonalization process is done at the level of MVAR coefficients [42]. The generalized version of OPDC by taking the effect of time series scaling into consideration is:

$$
\begin{aligned}
& \tilde{\Psi}_{p, q}(n, f)= \\
& \frac{1}{\lambda_{p, p}^{2}} \frac{\left|\operatorname{Real}\left\{A_{p, q}(n, f)\right\}\right|}{\sqrt{a_{q}^{\mathrm{H}}(n, f) \Sigma_{w}^{-1} a_{q}(n, f)}} \frac{\left|\operatorname{Imag}\left\{A_{p, q}(n, f)\right\}\right|}{\sqrt{a_{q}^{\mathrm{H}}(n, f) \Sigma_{w}^{-1} a_{q}(n, f)}} \text { if } p \neq q
\end{aligned}
$$

where $\lambda$ are the diagonal elements of covariance matrix $\Sigma_{w}=\left\langle w w^{p^{1}}\right\rangle$ (where $\langle\cdot\rangle$ is the expected value operator), the superscript $\mathrm{H}$ denotes the Hermitian operator and $a_{q}(n, f)$ is the $q$ th column of $A(n, f)$. For detailed mathematical derivation of gOPDC, please refer to [38].

\subsection{Effective Connectivity of microFE-EEGs}

To assess the particularity of brain connectivity under microFEs, effective connectivity with the resting-state-EEGs was firstly computed as a standard. Such resting-state threshold was calculated as the $99^{\text {th }}$ percentile gOPDC to eliminate outliers and form a connectivity baseline. By comparing with the threshold, only higher gOPDCs were retained to emphasize the stronger connectives. To reduce the computation, EEGs were down-sampled to $512 \mathrm{~Hz}$.

Focusing on the typical EEGs frequency range, gOPDCs were averaged into three representative bands as low 
frequency $[4 \mathrm{~Hz}, 12 \mathrm{~Hz})$, medium frequency $[12 \mathrm{~Hz}, 30 \mathrm{~Hz}$ ) and high frequency [30 Hz, $50 \mathrm{~Hz}$ ) [43]. Fig. 5 illustrates the normalized microFE-averaged connectivity of three EEG bands. Among three bands, high frequency shows the strongest connectivity. Among all channels, electrodes arranged on the motor cortex show relatively more information exchange than others (where ID.12-14 Cz \& C3 \& C4 show more information outflow and ID.19-20 CP5 \& CP6 show more information inflow).

Focusing on the dynamic process of microFEs, gOPDCs during the first $0.5 \mathrm{~s}$ (from $7.00 \mathrm{~s}$ to $7.50 \mathrm{~s}$ in each trial), the middle $0.5 \mathrm{~s}$ (from $8.75 \mathrm{~s}$ to $9.25 \mathrm{~s}$ ) and the last $0.5 \mathrm{~s}$ (from $10.50 \mathrm{~s}$ to $11.00 \mathrm{~s}$ ) were detailly assessed to reveal the connectivity changes from the very beginning to the end. Directed information flows with gOPDCs higher than other $90^{\text {th }}$ percentile were marked in Fig. 6, along with the channels performing more information interactions.

Among all 30 channels, those on and surround the motor cortex show more involvements and interactions. Typically, in micro-Left-Smirk, more interactions flow out of

\begin{tabular}{|l|l|l|l|}
\hline micro-Raise-Brow & micro-Furrow-Brow & micro-Left-Smirk & micro-Right-Smirk \\
\hline
\end{tabular}
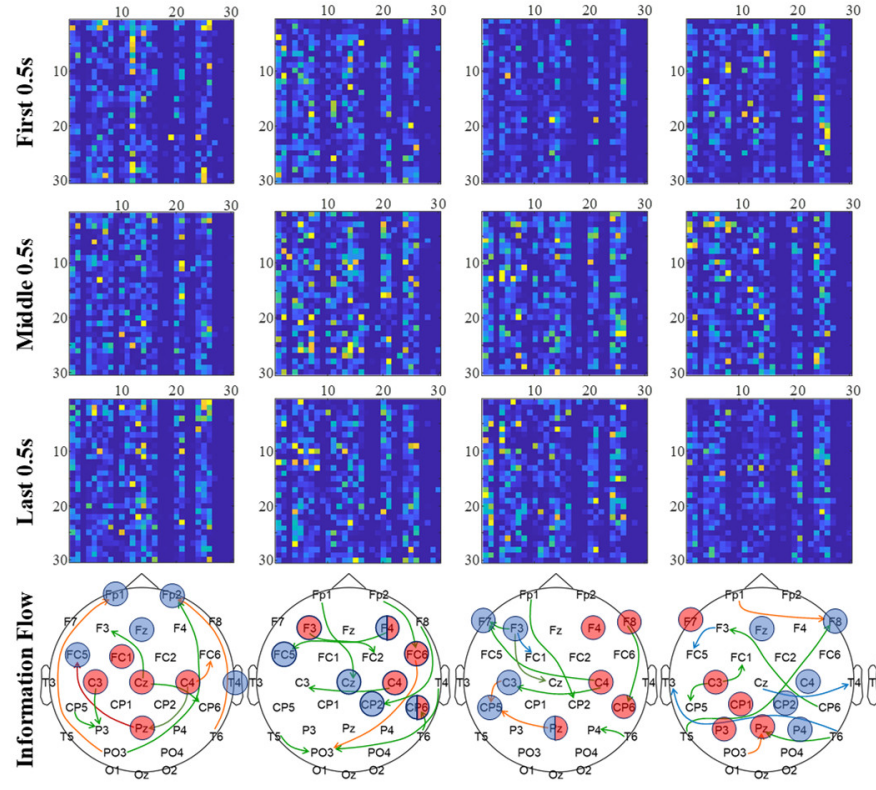

the right area into the left area of the brains; while in microRight-Smirk, more from the right to the left. The participation of the visual cortex may result from the UI prompt. Some slight asymmetry between the channels on the left and the right regions may be caused by the majority of right-handed.

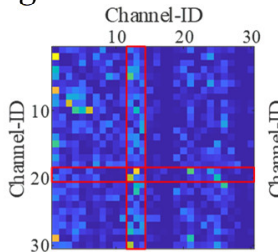

(a) Low frequency

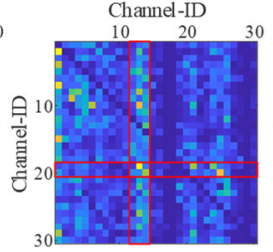

(b) Medium frequency

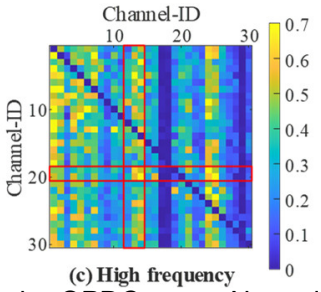

(c) High frequency 0 threshold of (a) low frequency, (b) medium frequency and (c) high frequency, where the value between the same channels were assigned as 'NaN', and the Channel ID.1 to ID.30 were listed as: Fp1, Fp2, Fz, F3, F4, F7, F8, FC1, FC2, FC5, FC6, Cz, C3, C4, T3, T4, CP1, CP2, CP5, CP6, Pz, P3, P4, T5, T6, PO3, PO4, Oz, 01 and O2. The block in ith row and jth column represents the gOPDC from Channel ID.j to Channel ID.i.

\section{micro-Smile $\quad$ micro-Open-Mouth $\mid$ micro-Pout-Mouth micro-Duck-Mouth}
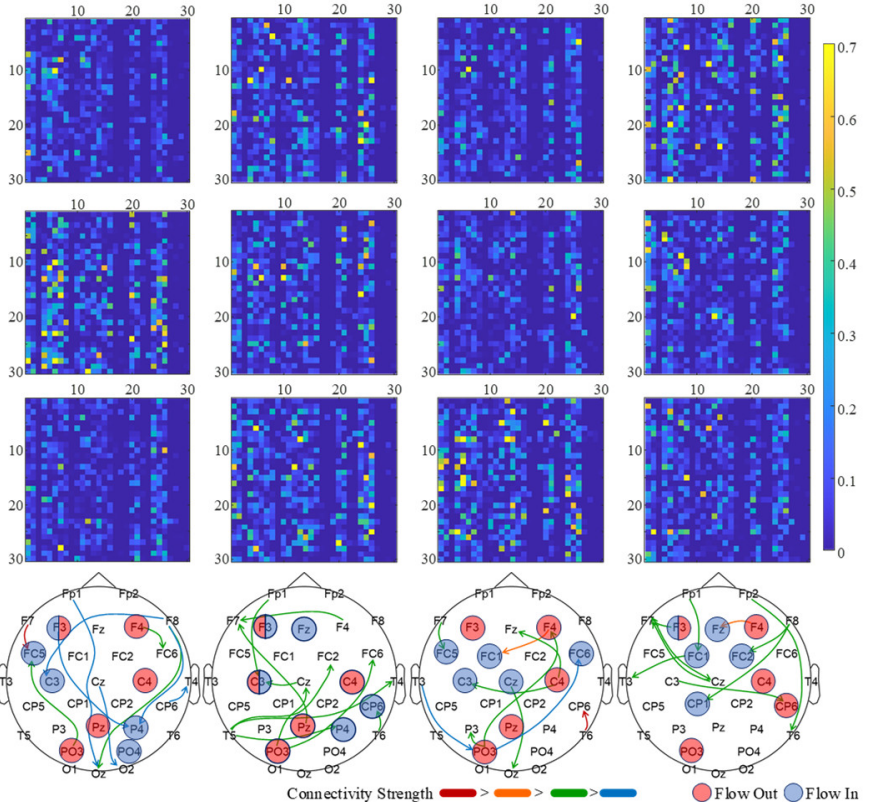

Fig. 6. The dynamic change of normalized subjects-averaged gOPDC during microFE, and the directed information flow with $90 \%$ percentile connectivity and channels with more interaction, where the list of Channel ID. 1 to ID.30 is the same as it in Fig. 5.

\section{MICROFE-BCI: ALGORITHM}

To meet the goal of practicality, the fundamental demands for one control approach are stability, accuracy, real-time, and user-friendliness. As for an EEG-based control paradigm, the specific technique details can be listed as: (1) Using fewer EEGs for each decoding, to improve the real-time ability; (2) Providing asynchronous strategy, to ensure users start or stop paradigm as needed; (3) Adopting a paradigm which requires no additional stimulators and adaptation, and has no strict requirement for high concentration; (4) Ensuring high decoding accuracy with adequate instruction set; (5) Giving an allowance to users' unrelated movements, without affecting the manipulation performance; (6) Reducing the common problem of misoperation, thus enhancing the stability.
In the real-time aspect, [44] stated that any delay greater than $200 \mathrm{~ms}$ would degrade the performance of one EEGbased task accomplishment. Considering the process of data acquisition, signal processing, and instruction generation, the EEGs window length used for once decoding in microFE-BCI is chosen as $100 \mathrm{~ms}$.

The processing strategy in microFE-BCI is demonstrated in Fig. 7. The algorithm can be divided into two stages as 'Asynchronous "ON" detection' and 'microFEBCI based real-time control', including four subdivided parts: (1) 'Is this probably microFE-EEGs or obvious 'NON", aiming at excluding obvious non-microFE-EEGs at the beginning; (2) 'Asynchronous interface detection', turning on or off the paradigm depends on users' will; (3) 'microFE-EEGs real-time decoding', classifying the microFE-EEGs and keep an extra 'NON' to give a respite for users, meanwhile a hold-on command for the system; (4) 
'Validity judgment', judging the validity of the label first to reduce misoperation instead of directly converting it into instruction.

Such step-by-step design is to shorten the processing time for EEGs unrelated to current system status (e.g., exaggerated facial-expression-EEGs would be rejected from the very beginning; EEGs would not be complexly classified until the asynchronous interface has been turned $\mathrm{ON}$ ), meanwhile offering an allowance for other slightly physical movements, and improving the stability. The detailed core algorithm is illustrated in Fig. 8.

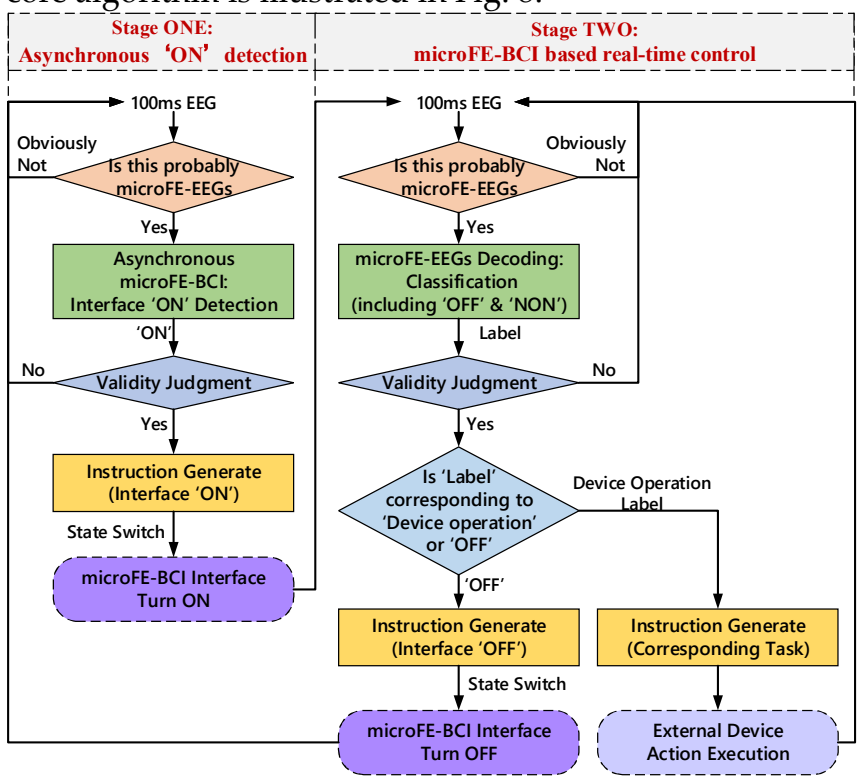

Fig. 7. The processing strategy in microFE-BCl

\subsection{Step A: Obvious non-microFE-EEGs exclusion}

The first step aims at using a simple computation to exclude EEGs that are apparently non-microFE-EEGs, while not let go of any probably microFE-EEGs. To save the computing time thus improving the efficiency, one junior method was chosen: Threshold Comparison.

Due to the EMG artifacts, when performing regular or exaggerated facial expressions, channels placed closer to facial muscles (Fp1, Fp2, F7, F8, T3, T4) tend to show wider amplitude range than microFEs, leading to larger temporal energy in higher EEG frequency [ $55 \mathrm{~Hz}, 95 \mathrm{~Hz}$ ], as shown in Fig. 9. These six channels were used as reference channels for obvious ' $N O N^{\prime}$ ' exclusion.

Instead of uniformly larger than one constant, the temporal energies are relatively larger within each reference channel itself and presents different distributions among microFEs and subjects. For lacking a uniform threshold, such Threshold Comparison was designed: When facing the latest unknown 100 ms EEGs, if there exist one or more channels whose temporal energy exceeds $105 \%$ of its maximum, the current EEGs would be judged as ' $\mathrm{NON}^{\prime}$ and be excluded. Those mistakenly-accepted EEGs, similar but not microFE-EEGs, would be processed by the next step.

\subsection{Step B: Interface 'ON' detection}

This step enables users to start the microFE-BCI whenever they need, which means system would not reply to any EEGs and keep a standby mode until the interface has been turned on. Such design guarantees users the option of not controlling the peripherals and handling other affairs $n$ with the EEG cap worn and all devices powered on.
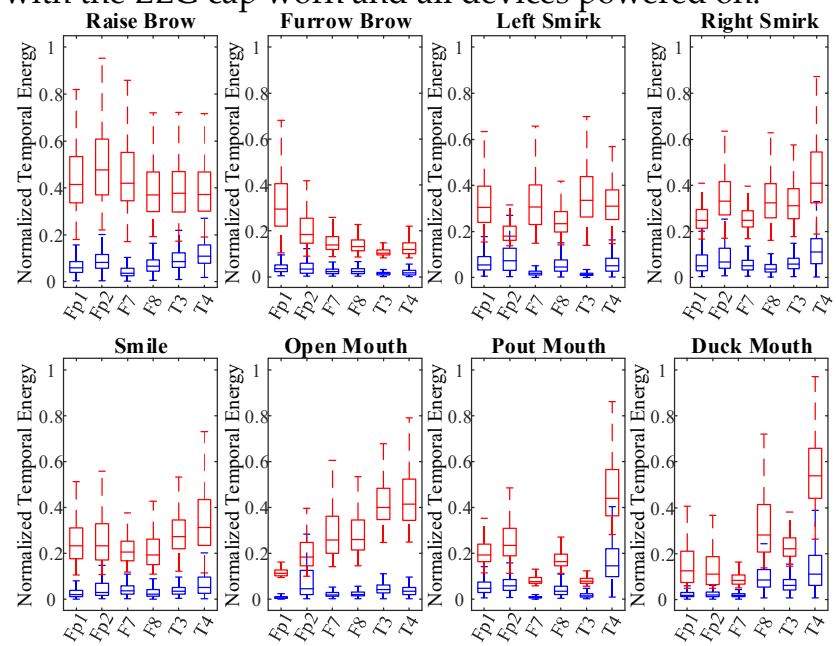

Fig. 9. The boxplot of subject S1's normalized temporal energy under microFE versus regular amplitude.

To switch between the non-control (NC) state and the in-control (IC) state, one of the eight microFEs was chosen as the state-switching microFE: micro-Raise-Brow. The decoding for asynchronous ' $\mathrm{ON}^{\prime}$ ' is realized by a binary classifier. Only micro-Raise-Brow-EEGs were labeled as ' $\mathrm{ON}^{\prime}$ ', while other EEGs including resting, relaxing, other microFEs, regular or exaggerated facial expressions, and EEGs mistakenly-accepted by Step $A$ were all labeled as 'else'. Instead of deep learning algorithms, one traditional machine learning algorithm was adopted to speed up the process: Support Vector Machine (SVM).

Considering of the low frequency solution resulted from the short time window, feature engineering was realized by a classic spatial filtering method: Common Spatial Pattern (CSP) [21]. The CSP features were calculated as:

$$
f_{k}=\frac{\log \left(\operatorname{var}\left(\mathrm{Y}_{i, k}^{j}\right)\right)}{\sum_{k=1}^{2 m} \log \left(\operatorname{var}\left(\mathrm{Y}_{i, k}^{j}\right)\right)}, k=1, \ldots, 2 m
$$

where $\mathrm{Y}_{i}^{j}, i \in\{1,2\}$ is the new signal generated through the spatial filters from raw data, in which $i$ indicates the $i^{\text {th }}$ category and $j$ indicates the $j^{\text {th }}$ sample, $k$ is the number of pairs of spatial filters and was set to be 2 in this paper.

Corresponding to pair number, a 4-dimensional feature vector was formed. The kernel function in SVM was set to be scaled gaussian (Equation (5)), according to the F1 score [45]. Seeing RESULTS for detailed comparison.

$$
\left\{\begin{array}{c}
\mathrm{G}\left(x_{j}, x_{k}\right)=\exp \left(-\gamma\left\|x_{j}-x_{k}\right\|^{2}\right) \\
\gamma=\frac{1}{n_{\text {feature }} * \operatorname{var}(\mathbf{X})}
\end{array}\right.
$$

where $\mathrm{G}\left(x_{j}, x_{k}\right)$ denotes elements in the Gram matrix, $\gamma$ is the hyperparameter for gaussian kernel, $\mathbf{X}$ is the features sets and $n_{\text {feature }}$ is the dimension of feature channels.

Every 100 ms EEGs that passed through Step A was refiltered into the typical EEG band as [ $5 \mathrm{~Hz}, 50 \mathrm{~Hz}]$ with all 30 channels and processed by this Step $B$. 


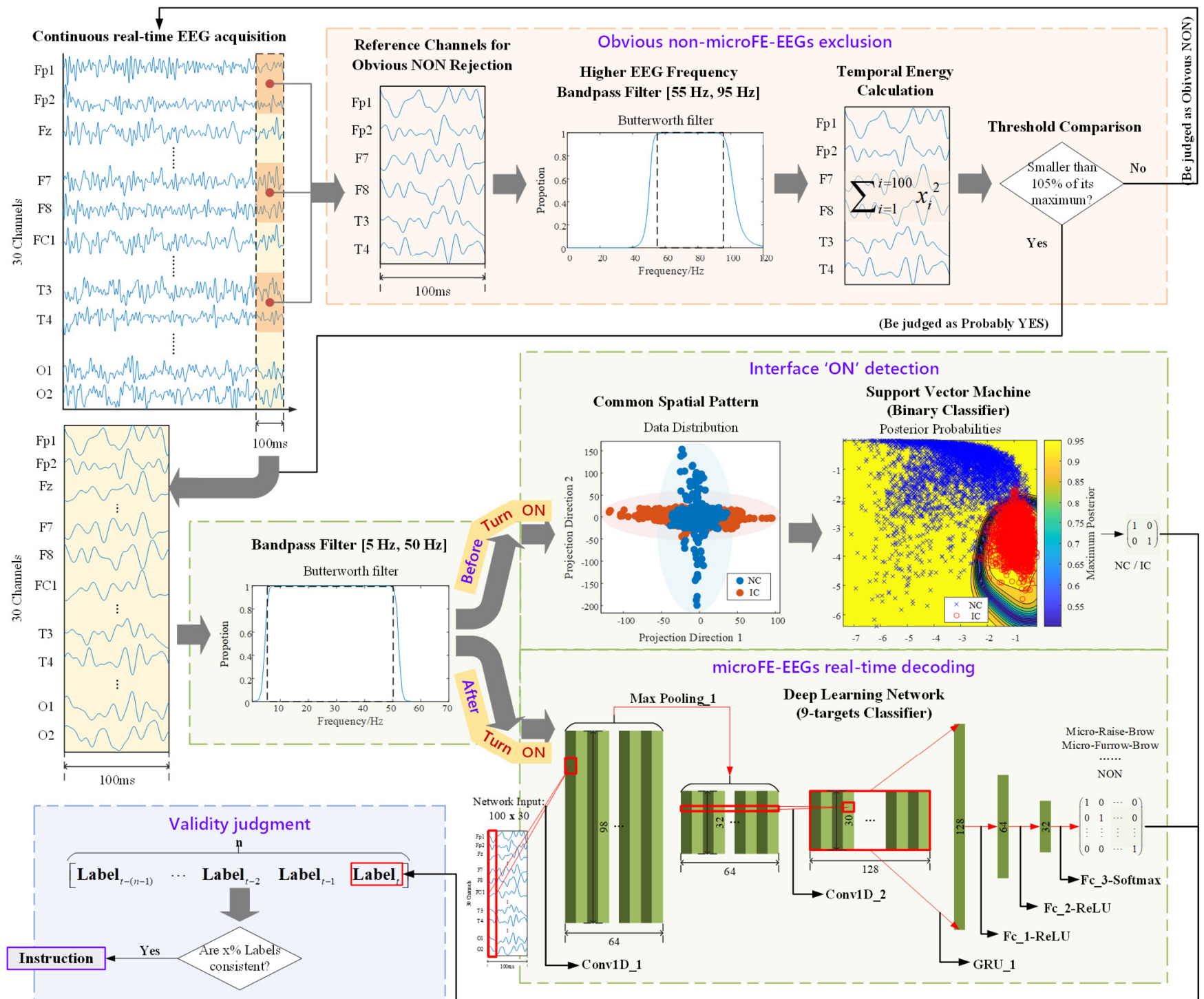

Fig. 8. The detailed core algorithm of microFE-BCl.

\subsection{Step C: microFE-EEGs real-time decoding}

After switching $\mathrm{ON}$ the microFE-BCI, the algorithm enters the $2^{\text {nd }}$ stage: microFE-BCI based real-time control. In the $2^{\text {nd }}$ stage, similarly, only those latest 100 ms EEGs accepted by Step $A$ can enter Step $C$. In this step, 9 targets were set in total, including: 7 microFEs corresponding to specified control instructions, the state-switching microFE corresponding to interface OFF, and a 'NON' category as the hold-on command for system. Instead of setting a separate asynchronous 'OFF' detection ahead, such design with the control instruction and interface OFF combined together, is to speed up the efficiency; Otherwise, any microFE-EEGs intend for control have to pass through two classifiers [25].

This multi-classification was realized by the deep-learning framework. To take fully advantage of the translation invariance and the spatial hierarchy of the Convolutional Neural Network (CNN) [46], meanwhile retaining the time sequential characteristics of EEGs, the variant of Recurrent Neural Network (RNN) [47] and the CNN were combined to form this classifier. The classifier structure (namely microFE-Net) was summarized in Table 1. In microFE-Net, one-dimensional Convolutional (Conv) layer along channels was adopted to extract the information at the same timestamp. Then, to lower the computational cost, the Gated Recurrent Unit (GRU) [48] layer was adopted instead of the Long Short-Term Memory (LSTM) [49]. To reduce the overfitting problem, the dropout and the recurrent dropout [50] were added within the GRU layer.

TABLE 1

SUMMARY OF MICROFE-NET

\begin{tabular}{|c|c|c|c|}
\hline Layer (type) & Method & Parameter & Value \\
\hline \multirow{3}{*}{ Conv1D_1 } & \multirow{3}{*}{$\begin{array}{l}\text { Temporal Di- } \\
\text { mension }\end{array}$} & Kernel Size & 3 \\
\hline & & Strides & 1 \\
\hline & & Filters & 64 \\
\hline \multirow{3}{*}{$\begin{array}{l}\text { Pool- } \\
\text { ing1D_1 }\end{array}$} & \multirow{3}{*}{ Max Pooling } & Pool Size & 3 \\
\hline & & Strides & 3 \\
\hline & & Padding & valid \\
\hline \multirow{3}{*}{ Conv1D_2 } & \multirow{3}{*}{$\begin{array}{l}\text { Temporal Di- } \\
\text { mension }\end{array}$} & Kernel Size & 3 \\
\hline & & Strides & 1 \\
\hline & & Filters & 128 \\
\hline \multirow{6}{*}{$\mathrm{GRU}_{-} 1$} & \multirow{6}{*}{---} & Activation & $\tanh$ \\
\hline & & Recurrent Activation & sigmoid \\
\hline & & Dropout & 0.1 \\
\hline & & Recurrent Dropout & 0.1 \\
\hline & & Return Sequences & false \\
\hline & & Units & 128 \\
\hline \multirow{2}{*}{ Dense } & \multirow{2}{*}{---} & Units & 64 \\
\hline & & Activation & ReLU \\
\hline
\end{tabular}




\begin{tabular}{cccc}
\hline Dense & --- & $\begin{array}{c}\text { Units } \\
\text { Activation }\end{array}$ & $\begin{array}{c}32 \\
\text { ReLU }\end{array}$ \\
\hline Dense & One Hot & $\begin{array}{c}\text { Units } \\
\text { Activation }\end{array}$ & $\begin{array}{c}9 \\
\text { Softmax }\end{array}$ \\
\hline $\begin{array}{c}\text { Loss Func- } \\
\text { tion }\end{array}$ & $\begin{array}{c}\text { Categorical } \\
\text { Cross Entropy }\end{array}$ & -- & -- \\
\hline Optimizer & RMSprop & Learning Rate & 0.001 \\
\hline $\begin{array}{c}\text { Training } \\
\text { Acceleration }\end{array}$ & Batch & Batch Size & 128 \\
\hline \hline
\end{tabular}

The input of microFE-Net was 30-channel EEGs with 100 sample points, which are judged by Step $A$ ahead as probably YES and re-filtered into [ $5 \mathrm{~Hz}, 50 \mathrm{~Hz}]$. The outputs of microFE-Net were 9 labels with one-hot code, which corresponding to 8 microFEs and a ' $\mathrm{NON}^{\prime}$.

\subsection{Step D: Validity judgment}

For realistic manipulation tasks, any misoperation shall result in a huge impact on the execution. To improve the robustness, instead of directly translating each decoded label into instruction, a Validity judgment was added between. Labels generated from Step B and Step C shall pass through Step $D$. Such judgment was simply realized by the consistency checking: Among $n$ decoded labels, if $x \%$ of them are consistent with the current $n^{\text {th }}$, then the $n^{\text {th }}$ will be regarded as valid and translated into an instruction.

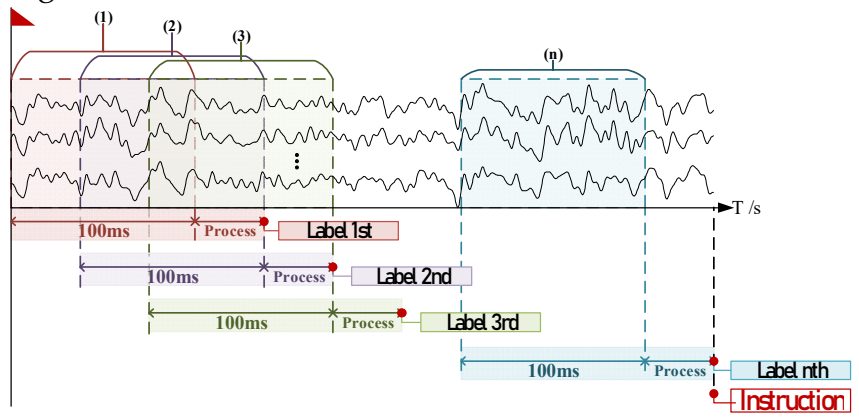

Fig. 10. The timing diagram for one valid instruction generation, supposed that all collected data were microFE-EEGs intending for the same label.

During processing, the timespan for each label's decoding is less than $100 \mathrm{~ms}$ (referring to Results), thus adjacent EEGs entering the algorithm should have an overlap. Giving that there exist $x \%$ decoded labels consistent with the current $n^{\text {th }}$, the timing diagram for instruction generation is exampled in Fig. 10. The theoretical timespan counting from the EEGs collection to the instruction generation is:

$$
\text { Ts }=100+n \times \text { ts }(\mathrm{ms})
$$

where Ts is the timespan of one instruction, ts is the timespan of one decoded label, and $n$ is set to be 20 in this paper comprehensively considering both the robustness and the rapidity.

\section{Results}

To determine the algorithm parameters and develop the microFE-BCI software, data collected in offline experiments from six subjects were used in the offline assessment first. Then, ten subjects participated in the online manipulation based on the microFE-BCI software to evaluate the practical ability of such EEG-based control paradigm. During the offline assessment, the accuracy, precision and time cost are given more consideration; While in the online evaluation, the controllability and the completion quality of tasks are more emphasized.

\subsection{Offline Datasets}

Data in offline expriment were sliced into pieces and stacked. The imbalance for training datasets (in Step B and Step C) was solved by overlap, as illustrated in Fig. 11.

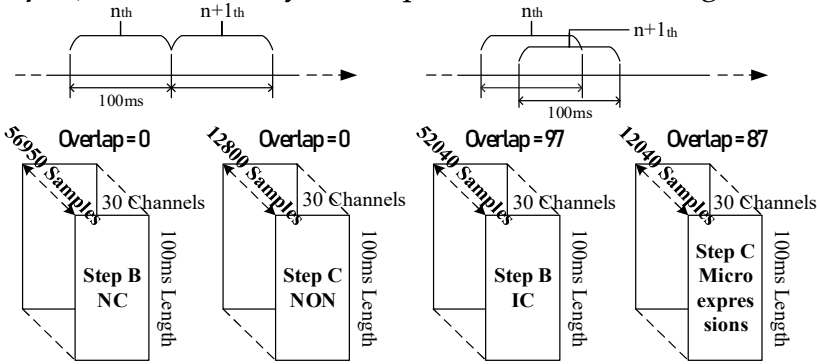

Fig. 11. The illustration for datasets in offline assessment.

Within Step B's training, the NC dataset consists of EEGs from resting-state, relaxing-state, 8 facial-expressions with regular amplitude, and 7 non-state-switching microFEs; While the IC dataset only generated from stateswitching microFE-EEGs. In Step $C^{\prime}$ s training, the dataset for ' $\mathrm{NON}^{\prime}$ ' was sliced from the resting-state EEG.

\subsection{Offline Assessment}

\section{- Step A: Obvious non-microFE-EEGs exclusion}

Compared to the former FE-BCI [21], by limiting the range of facial muscle movement to the micro level, this microFE$\mathrm{BCI}$ greatly reduces the proportion of EMG artifacts and makes it possible to distinguish between daily facial-expressions and microexpressions in EEG-based control. The contrast of EMG artifacts proportion is particularly obvious in the high frequency of EEG. Fig. 12 shows the comparison between regular facial-expression-EEGs versus microFE-EEGs.
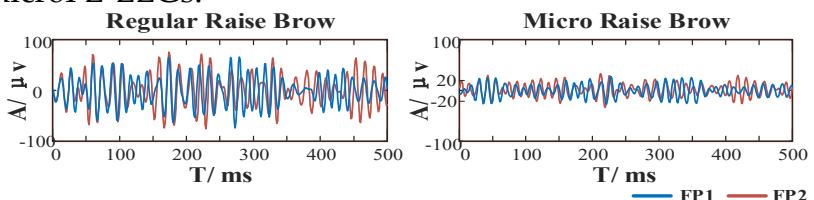

Fig. 12. Comparison between EEGs when performing regular-RaiseBrow versus. micro-Raise-Brow in $[55 \mathrm{~Hz}, 95 \mathrm{~Hz}]$ of channel FP1 \& FP2.

In Fig. 12, the amplitude range of microFE-EEGs is much smaller than that under regular facial-expressions. To exclude non-microFE-EEGs, several different methods for threshold setting are compared in Table 2.

$$
\text { TABLE } 2
$$

COMPARISON AMONG DifFERENT THRESHOLd SETTING METHOdS

\begin{tabular}{cccccc}
\hline \hline Method & Formula & $\begin{array}{c}\text { Acceptance } \\
\text { Rate } \\
(\mathrm{AR})\end{array}$ & $\begin{array}{c}\text { Rejection } \\
\text { Rate } \\
(\mathrm{RR})\end{array}$ & $\begin{array}{c}\text { Timespan } \\
(\mathrm{Ts})\end{array}$ & Score \\
\hline Maximum & $\begin{array}{l}\max \left\{\left|x_{i}\right|\right\}, \\
(i=1, \ldots, 100)\end{array}$ & $99.48 \%$ & $64.67 \%$ & $0.0055 \mathrm{~ms}$ & 39.11 \\
$\begin{array}{c}\text { Temporal } \\
\text { Energy }\end{array}$ & $\sum_{i=1}^{i=100} x_{i}^{2}$ & $99.45 \%$ & $68.66 \%$ & $0.0055 \mathrm{~ms}$ & 40.53 \\
$\begin{array}{c}\text { Amplitude } \\
\text { Range }\end{array}$ & $\begin{array}{c}\max \left\{x_{i}\right\}-\min \left\{x_{i}\right\} \\
(i=1, \ldots, 100)\end{array}$ & $99.48 \%$ & $64.80 \%$ & $0.0055 \mathrm{~ms}$ & 39.15 \\
\hline \hline
\end{tabular}

* The score is calculated as $1 /(1 / \mathrm{AR}+1 / \mathrm{RR}+\mathrm{Ts})$

Throughout the three methods, the temporal energy achieves the highest score. By setting the threshold as temporal energy, the correctly acceptance rates (ARs) of 
different microFEs are listed in Table 3.

ARs in Table 3 are nearly $99 \%$ and shows no typical imbalances. The extremely short timespan, high AR, and high RR prove the feasibility of threshold comparison as the first segment of the algorithm.

TABLE 3

CORRECTLY ACCEPTANCE RATE OF 8 MICROFES WITH THRESHOLD AS TEMPORAL ENERGY

$\begin{array}{lllllll}\mathrm{m}- & \mathrm{m}- & \mathrm{m}- & \mathrm{m}- & \mathrm{m}- & \mathrm{m}- & \mathrm{m}-\mathrm{m}-\end{array}$

$\begin{array}{ccccccccc}\text { S } & \text { RB } & \text { FB } & \text { LS } & \text { RS } & \text { OM } & \text { S } & \text { PM } & \text { DM }\end{array}$

$\begin{array}{lllllllll}\text { S1 } & 97.19 \% & 100 \% & 99.94 \% & 99.25 \% & 100 \% & 99.31 \% & 99.56 \% & 99.94 \%\end{array}$

S2 $\quad 98.38 \% \quad \begin{array}{llllllll}98.88 \% & 99.75 \% & 100 \% & 99.94 \% & 99.06 \% & 99.94 \% & 99.25 \%\end{array}$

$\begin{array}{lllllllll}\text { S3 } & 98.63 \% & 100 \% & 98.81 \% & 98.81 \% & 100 \% & 100 \% & 100 \% & 100 \%\end{array}$

$\begin{array}{lllllllll}\text { S4 } & 98.06 \% & 99.94 \% & 98.88 \% & 99.75 \% & 100 \% & 99.06 \% & 99.94 \% & 100 \%\end{array}$

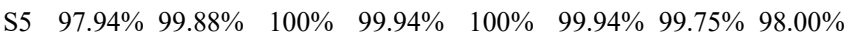
$\begin{array}{lllllllll}\text { S6 } & 97.75 \% & 100 \% & 99.00 \% & 99.13 \% & 99.88 \% & 100 \% & 99.94 \% & 100 \%\end{array}$

* $m$-RB stands for micro-Raise-Brow, $m$-FB for micro-Furrow-Brow, $m$-LS for micro-Left-Smirk, m-RS for micro-Right-Smirk, m-OM for micro-OpenMouth, $m$-Sl for micro-Smile, $m$-PM for micro-Pout-Mouth, $m$-DM for micro-Duck-Mouth

\section{- Step B: Interface ‘ON' detection}

To select one microFE as the most suitable microFE for state-switching, 8 microFEs were first compared using SVMs with 4 different kernel functions, as listed in Table 4 .

TABLE 4

THE ACCURACY FOR 8 DIFFERENT STATE-SWITCHING-MICROFES

\begin{tabular}{ccccc}
\hline \multirow{2}{*}{ MicroFE } & \multicolumn{4}{c}{ SVM kernel } \\
\cline { 2 - 5 } & $\begin{array}{c}\text { Linear } \\
\text { Polynomial }\end{array}$ & $\begin{array}{c}\text { Quadratic } \\
\text { Polynomial }\end{array}$ & $\begin{array}{c}\text { Scaled } \\
\text { Gaussian }\end{array}$ & $\begin{array}{c}\text { Medium } \\
\text { Gaussian }\end{array}$ \\
\hline micro-Raise-Brow & $96.19 \%$ & $74.59 \%$ & $96.46 \%$ & $96.30 \%$ \\
micro-Furrow-Brow & $80.90 \%$ & $50.36 \%$ & $84.07 \%$ & $83.28 \%$ \\
micro-Left-Smirk & $76.70 \%$ & $51.54 \%$ & $81.08 \%$ & $80.60 \%$ \\
micro-Right-Smirk & $80.23 \%$ & $52.85 \%$ & $83.33 \%$ & $82.75 \%$ \\
micro-Open-Mouth & $72.84 \%$ & $53.28 \%$ & $81.66 \%$ & $81.41 \%$ \\
micro-Smile & $71.49 \%$ & $50.23 \%$ & $78.37 \%$ & $77.27 \%$ \\
micro-Pout-Mouth & $68.95 \%$ & $49.55 \%$ & $75.32 \%$ & $74.68 \%$ \\
micro-Duck-Mouth & $66.95 \%$ & $49.14 \%$ & $77.36 \%$ & $76.76 \%$ \\
\hline \hline
\end{tabular}

In Tabel 4, micro-Raise-Brow achieves the highest overall accuracy when be set as the state-switching microFE. The detailed performances for SVM kernels are listed in Table 5, with the micro-Raise-Brow as the state-switching microFE. Additionally, the time cost of CSP feature engineering is $0.1328 \mathrm{~ms}$ in average.

$$
\text { TABLE } 5
$$

THe DetaILED PERFormance OF DIFFERENT SVM KeRneLS WITH MICRO-RAISE-BROW AS STATE-SWITCHING MICROFE

\begin{tabular}{ccccccc}
\hline \hline $\begin{array}{c}\text { SVM } \\
\text { kernel }\end{array}$ & Accuracy Precision & Recall & $\begin{array}{c}\mathrm{F} 1 \\
\text { Score }\end{array}$ & $\begin{array}{c}\text { Prediction } \\
\text { Speed }\end{array}$ & $\begin{array}{c}\text { Training } \\
\text { Duration }\end{array}$ \\
\hline $\begin{array}{c}\text { Linear } \\
\text { Polyno- } \\
\text { mial }\end{array}$ & $96.19 \%$ & $94.58 \%$ & $98.02 \%$ & 48.14 & $490000 \mathrm{obs} / \mathrm{sec}$ & $>15 \mathrm{~min}$ \\
$\begin{array}{c}\text { Quadratic } \\
\text { Polyno- } \\
\text { mial }\end{array}$ & $74.59 \%$ & $75.62 \%$ & $73.23 \%$ & 37.20 & $96000 \mathrm{obs} / \mathrm{sec}$ & $>15 \mathrm{~min}$ \\
$\begin{array}{c}\text { Scaled } \\
\text { Gaussian } \\
\text { Medium } \\
\text { Gaussian }\end{array}$ & $96.46 \%$ & $95.29 \%$ & $97.78 \%$ & 48.26 & $130000 \mathrm{obs} / \mathrm{sec}$ & $<3 \mathrm{~min}$ \\
\hline \hline
\end{tabular}

* Evaluation with Win10, i5-6500, 3.20GHz, without GPU accelerated.

Among 4 SVM kernels, the linear polynomial, the scaled aussian, and the medium gaussian achieve higher F1 scores [45]. Comprehensively considering the F1 score, prediction speed, and training duration, the scaled gaussian performs best. The adaptability of scaled gaussian kernel to each subject is listed in Table 6.

TABLE 6

The ACCURACy, PRECISION AND RECALL WITH SCALEd Gaussian

\begin{tabular}{cccc}
\hline \hline S & Accuracy & Precision & Recall \\
\hline S1 & $97.79 \%$ & $96.14 \%$ & $99.57 \%$ \\
S2 & $96.47 \%$ & $95.22 \%$ & $97.85 \%$ \\
S3 & $96.62 \%$ & $97.09 \%$ & $96.13 \%$ \\
S4 & $94.60 \%$ & $92.50 \%$ & $97.06 \%$ \\
S5 & $97.11 \%$ & $95.65 \%$ & $98.70 \%$ \\
S6 & $96.18 \%$ & $95.13 \%$ & $97.34 \%$ \\
\hline Mean & $96.46 \%$ & $95.29 \%$ & $97.78 \%$ \\
Std & \pm 1.07 & \pm 1.54 & \pm 1.22 \\
\hline \hline
\end{tabular}

The accuracy, precision, and recall in Table 6 show a relatively small standard deviation (Std) among subjects. Based on all the assessments above, the CSP combining with scaled-gaussian-SVM proves its best applicability, which maintains a stable performance without obvious individual differences.

\section{- Step C: microFE-EEGs real-time decoding}

As a multi-classification with 9 targets based on deeplearning, several mature structures are assessed to verify their applicability first, such as VGG16 [51], DenseNet121 [52] and ResNet50 [53]. To match with this specified problem, all network applications were imported without pretrained weights and slightly modified, as shown in Table 7. Compared with the microFE-Net, their accuracies under 10 -fold validation are listed in Table 8 .

TABLE 7

STRUCTURE FOR NETWORK APPLICATIONS

\begin{tabular}{|c|c|c|c|}
\hline Layer (type) & Method & Parameter & Value \\
\hline \multirow{2}{*}{$\begin{array}{c}\text { Network } \\
\text { Applications }\end{array}$} & \multirow[b]{2}{*}{---} & Pretrained weights & None \\
\hline & & Top layer & Not include \\
\hline Dense & --- & $\begin{array}{c}\text { Units } \\
\text { Activation }\end{array}$ & $\begin{array}{c}256 \\
\text { ReLU }\end{array}$ \\
\hline Dropout & --- & ---- & 0.5 \\
\hline Dense & One Hot & $\begin{array}{c}\text { Units } \\
\text { Activation }\end{array}$ & $\begin{array}{c}9 \\
\text { Softmax }\end{array}$ \\
\hline $\begin{array}{l}\text { Loss Func- } \\
\text { tion }\end{array}$ & $\begin{array}{c}\text { Categorical } \\
\text { Cross Entropy }\end{array}$ & --- & --- \\
\hline Optimizer & RMSprop & Learning Rate & 0.001 \\
\hline $\begin{array}{c}\text { Training } \\
\text { Acceleration }\end{array}$ & Batch & Batch Size & 128 \\
\hline
\end{tabular}

TABLE 8

Performance Comparison AMONg DeEP-LEARning Structures

\begin{tabular}{ccc}
\hline \hline Deep Learning Network & Training Accuracy & Validation Accuracy \\
\hline VGG16 & $11.03 \%$ & $11.34 \%$ \\
DenseNet121 & $98.91 \%$ & $88.94 \%$ \\
ResNet50 & $98.52 \%$ & $87.27 \%$ \\
microFE-Net & $92.68 \%$ & $89.48 \%$ \\
\hline
\end{tabular}

Through comparision, DenseNet121 and ResNet50 both achieve higher training accuracy, but also face serious overfitting problems (even would suddenly drop to a $40 \%$ validation accuracy in the late-training-process). Compared with 3 mature network applications, the microFE-Net, although not the highest training accuracy, it shows a more balanced performance between training and validation.

In addition to the network structure, extra feature engineering steps were combined to assess its effectiveness (Table 9). For the microFE-Net model isn't designed to accept 
4-dimensional input tensors, the classifier combined with the Filter Bank Common Spatial Pattern (FB-CSP) [54] feature engineering was selected to be the DenseNet121.

TABLE 9

Performance Comparison for EXTRA FEATURE ENGINEERING

\begin{tabular}{ccccc}
\hline \hline Method & Feature Engineering & Classifier & $\begin{array}{c}\text { Training } \\
\text { Accuracy }\end{array}$ & $\begin{array}{c}\text { Validation } \\
\text { Accuracy }\end{array}$ \\
\hline \multirow{2}{*}{$\mathrm{A}$} & CSP & SVM & $74.24 \%$ & $71.45 \%$ \\
& (One vs Rest) & (Fine Gaussian) & & \\
$\mathrm{B}$ & FB-CSP & DenseNet121 & $89.87 \%$ & $36.63 \%$ \\
$\mathrm{C}$ & (3 Frequency Bands) & microFE-Net & $92.68 \%$ & $89.48 \%$ \\
\hline \hline
\end{tabular}

In Table 9, the microFE-Net without any hand-made feature engineering achieves the best performance. The iteration of microFE-Net is shown in Fig. 13. The detailed accuracy of microFE-Net under 10 -fold validation and its prediction speed were listed in Table 10.

As demonstrated in Table 10, the microFE-Net achieves a stable performance varying among subjects with a $\pm 1.21 \%$ Std; Meanwhile maintains a precise accuracy and relatively small overfitting (with the validation accuracy as $89.48 \%$ and the training accuracy as $92.68 \%$ ). The averaged time cost for one prediction is nearly $0.235 \mathrm{~ms}$. The confusion matrix among 8 microFEs, in other words, 7 control targets and 1 state-switching target is listed in Table 11.

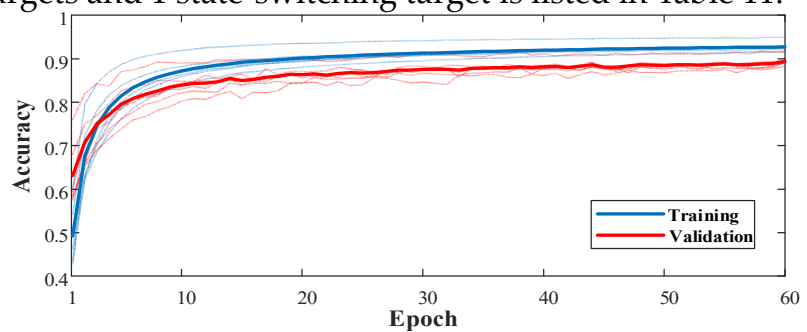

Fig. 13. The learning process of microFE-Net.

TABLE 10

OFFLINE ACCURACY OF MICROFE-NET WITH 10-FOLD VALIDATION

\begin{tabular}{cccc}
\hline \hline S & Training Accuracy & Validation Accuracy & Prediction Speed \\
\hline S1 & $92.58 \%$ & $89.59 \%$ & $256000 \mathrm{obs} / \mathrm{sec}$ \\
S2 & $92.45 \%$ & $89.06 \%$ & $256000 \mathrm{obs} / \mathrm{sec}$ \\
S3 & $91.21 \%$ & $88.00 \%$ & $257000 \mathrm{obs} / \mathrm{sec}$ \\
S4 & $92.25 \%$ & $89.34 \%$ & $254000 \mathrm{obs} / \mathrm{sec}$ \\
S5 & $94.89 \%$ & $91.69 \%$ & $254000 \mathrm{obs} / \mathrm{sec}$ \\
S6 & $92.69 \%$ & $89.17 \%$ & $256000 \mathrm{obs} / \mathrm{sec}$ \\
\hline Mean & $92.68 \%$ & $89.48 \%$ & -- \\
Std & \pm 1.21 & \pm 1.21 & -- \\
\hline \hline
\end{tabular}

* Evaluation with Win10, i5-6500, 3.20GHz, accelerated with GTX 960.

TABLE 11

CONFUSION MATRIX AMONG 8 MICROFES

\begin{tabular}{|c|c|c|c|c|c|c|c|c|c|}
\hline \multirow{2}{*}{ True Label } & \multicolumn{9}{|c|}{ Predicted Label } \\
\hline & $\mathrm{m}-\mathrm{RB}$ & $\mathrm{m}-\mathrm{FB}$ & m-LS & $\mathrm{m}-\mathrm{RS}$ & $\mathrm{m}-\mathrm{OM}$ & m-DS & $\mathrm{m}-\mathrm{PM}$ & $\mathrm{m}-\mathrm{DM}$ & NON \\
\hline $\mathrm{m}-\mathrm{RB}$ & $99.15 \%$ & $0.07 \%$ & $0.00 \%$ & $0.05 \%$ & $0.05 \%$ & $0.01 \%$ & $0.01 \%$ & $0.03 \%$ & $0.63 \%$ \\
\hline $\mathrm{m}-\mathrm{FB}$ & $0.08 \%$ & $94.91 \%$ & $0.63 \%$ & $0.48 \%$ & $0.44 \%$ & $0.27 \%$ & $0.21 \%$ & $0.05 \%$ & $2.93 \%$ \\
\hline $\mathrm{m}-\mathrm{LS}$ & $0.03 \%$ & $1.02 \%$ & $92.17 \%$ & $0.51 \%$ & $0.39 \%$ & $1.12 \%$ & $0.33 \%$ & $0.21 \%$ & $4.23 \%$ \\
\hline $\mathrm{m}-\mathrm{RS}$ & $0.05 \%$ & $0.22 \%$ & $0.78 \%$ & $94.05 \%$ & $0.20 \%$ & $1.38 \%$ & $0.20 \%$ & $0.27 \%$ & $2.85 \%$ \\
\hline $\mathrm{m}-\mathrm{OM}$ & $0.10 \%$ & $0.39 \%$ & $0.25 \%$ & $0.18 \%$ & $93.23 \%$ & $0.22 \%$ & $0.82 \%$ & $0.66 \%$ & $4.14 \%$ \\
\hline m-DS & $0.07 \%$ & $0.13 \%$ & $0.99 \%$ & $0.83 \%$ & $0.27 \%$ & $93.20 \%$ & $0.96 \%$ & $0.92 \%$ & $2.63 \%$ \\
\hline m-PM & $0.09 \%$ & $0.23 \%$ & $0.43 \%$ & $0.16 \%$ & $1.07 \%$ & $1.24 \%$ & $91.61 \%$ & $0.90 \%$ & $4.27 \%$ \\
\hline m-DM & $0.05 \%$ & $0.13 \%$ & $0.48 \%$ & $0.36 \%$ & $1.07 \%$ & $1.15 \%$ & $1.05 \%$ & $90.20 \%$ & $5.51 \%$ \\
\hline
\end{tabular}

Among the 8 microFEs, the micro-Raise-Brow (corresponding to state-switching) achieves the highest prediction accuracy as $99.15 \%$; While the lowest is micro-DuckMouth as $90.20 \%$. The averaged prediction accuracy among such 8 microFEs is $93.56 \%$. In each true label, the confusion with 'NON' is especially higher than the others, which decreases the averaged classification accuracy. The high misrecognition rate between the true label and ' $N O N^{\prime}$ may cause by the small change between the microFEs and the resting-state, which results in similar signal ingredients. Considering the advantage to retain the ' $\mathrm{NON}$ ' label as a hold-on command, which provides the system a rest even after entering the IC state, such 'NON' label cannot be deleted and is designed to be remained. The stability of the final instruction is enhanced by Step $D$ to improve accuracy.

\section{- Step D: Validity judgment}

As been detailly assessed above, the timespan (with ample margin) of each step is summarized in Table 12.

The theoretical timespans for different instructions are all less than $200 \mathrm{~ms}$, which meet the specified requirement for real-time control. Considering the various classification accuracy for different targets, the timespan for generating one specific instruction under realistic operation can only be assessed during online.

TABLE 12

TIMESPAN (WITH AMPLE MARGIN) OF EACH STEP

\begin{tabular}{ll}
\hline \hline \multicolumn{1}{c}{ Step } & Timespan \\
\hline Step A: Obvious non-microFE-EEGs exclusion & $\approx 0.01 \mathrm{~ms}$ \\
Step $B$ : Interface 'ON' detection & $\approx 0.15 \mathrm{~ms}$ \\
Step C: microFE-EEGs real-time decoding & $\approx 0.24 \mathrm{~ms}$ \\
\hline \hline
\end{tabular}

According to the theoretical time cost for generating one instruction in (6), the timespan for different instruction is listed in Tab. 13.

TABLE 13

THEORETICAL TIMESPAN FOR INSTRUCTION GENERATION

\begin{tabular}{cc}
\hline \hline THEORETICAL TIMESPAN FOR INSTRUCTION GENERATION \\
\hline Instruction & Theoretical Timespan \\
\hline Asynchronous interface 'ON' & $\approx 103.2 \mathrm{~ms}$ \\
Asynchronous interface 'OFF' & $\approx 105.0 \mathrm{~ms}$ \\
microFE-BCI based control instruction & $\approx 105.5 \mathrm{~ms}$ \\
\hline \hline
\end{tabular}

\subsection{Online Evaluation}

Based on the offline assessment, a microFE-BCI software has been developed to facilitate the easy application for the online external device control. This software includes three modules: 'user login', 'data collection and offline learning', and 'online application'. By using this software, the online microFE-BCI controlling task with AUBO-i5 robotic arm 
system and the 2-DoFs prosthetic hand were conducted.

In the 'online application' module, a 'debug' mode is provided to adjust the consistency checking criteria by subject-selves, without peripherals connected. Until the accuracy of valid instruction reached approximately a stable $100 \%$, then the online manipulation experiment would start with the machine connected.

Within the stepping control strategy adopted by these two peripherals, the stepping distances are not elaborately designed for tasks. Due to the visual perspective, stepping distance and some unavoidable random errors, these tasks can hardly be completed with perfect zero deviation, even by using the referred control method (i.e., FlexPendant and Joystick). In the following online evaluation, the practicality and the controllability of the microFE-BCI are of more concerned (e.g., the floating range of the indicator) than the precision. Compared between these two tasks, Task one requires higher stability, while Task two emphasized more on agility and real-time ability.

\section{- Task one: Object-moving with a Robotic Arm}

To manipulate the AUBO-i5 robotic arm system, all $8 \mathrm{mi}-$ croFEs were enabled. The instruction list is listed in Table 14, where the direction for axis $X, Y$, and $Z$ is previously marked in Fig. 4(a).

Considering the different important levels of instructions (e.g., a trial would be judged as a failure once the electric gripper is mistakenly opened halfway), the microFE with the highest recognition accuracy (except for the
micro-Raise-Brow) is assigned to the most vital instruction, differing from each subject. As an example, the correspondence between the microFEs and the system instruction of subject S7 is listed in Table 15, where micro-RaiseBrow is responsible for the state-switching as mentioned before. Table 15 also lists the consistency checking criteria $x \%$ for subject $\mathrm{S} 7$, which is personally tuned under 'debug' mode. Fig. 14 demonstrates the time log of a successful online manipulation for Task one.

\section{TABLE 14}

THE INSTRUCTION LIST OF AUBO-15 ROBOTIC ARM SYSTEM

\begin{tabular}{lccc}
\hline \hline Instruction & System Action & Axis & Stepping \\
\hline 'Right' & Horizontally move right & $+\mathrm{X}$ & $30 \mathrm{~mm}$ \\
'Left' & Horizontally move left & $-\mathrm{X}$ & $2 \mathrm{~mm}$ \\
'Forward' & Horizontally move forward & $+\mathrm{Y}$ & $20 \mathrm{~mm}$ \\
'Back- & Horizontally move backward & $-\mathrm{Y}$ & $3 \mathrm{~mm}$ \\
ward' & Vertically rise up & $+\mathrm{Z}$ & $30 \mathrm{~mm}$ \\
'Up' & Vertically lower down & $-\mathrm{Z}$ & $\left\{\begin{array}{l}10 \mathrm{~mm}(\mathrm{Z}>21 \mathrm{~mm}) \\
3 \mathrm{~mm}(\mathrm{Z} \leq 21 \mathrm{~mm}) \\
\text { 'Down' }\end{array}\right.$ \\
'Open' & Electric gripper open & -- & -- \\
\hline \hline
\end{tabular}

TABLE 15

The Operational CorRespondence, Along With THE Consistency CHECKING CRITERIA $x \%$ FOR SUBJECT S7 IN TASK ONE

\begin{tabular}{ccccccccc}
\hline \hline \multirow{2}{*}{ Item } & $\mathrm{m}-$ & $\mathrm{m}-$ & $\mathrm{m}-$ & $\mathrm{m}-$ & $\mathrm{m}-$ & $\mathrm{m}-$ & $\mathrm{m}-$ & $\mathrm{m}-$ \\
& $\mathrm{RB}$ & $\mathrm{FB}$ & LS & RS & OM & $\mathrm{S}$ & $\mathrm{PM}$ & $\mathrm{DM}$ \\
\hline Instruction & -- & Open & Left & Right & Up & Down & Backward & Forward \\
Criteria & 0.95 & 0.95 & 0.35 & 0.45 & 0.45 & 0.95 & 0.55 & 0.80 \\
\hline \hline
\end{tabular}

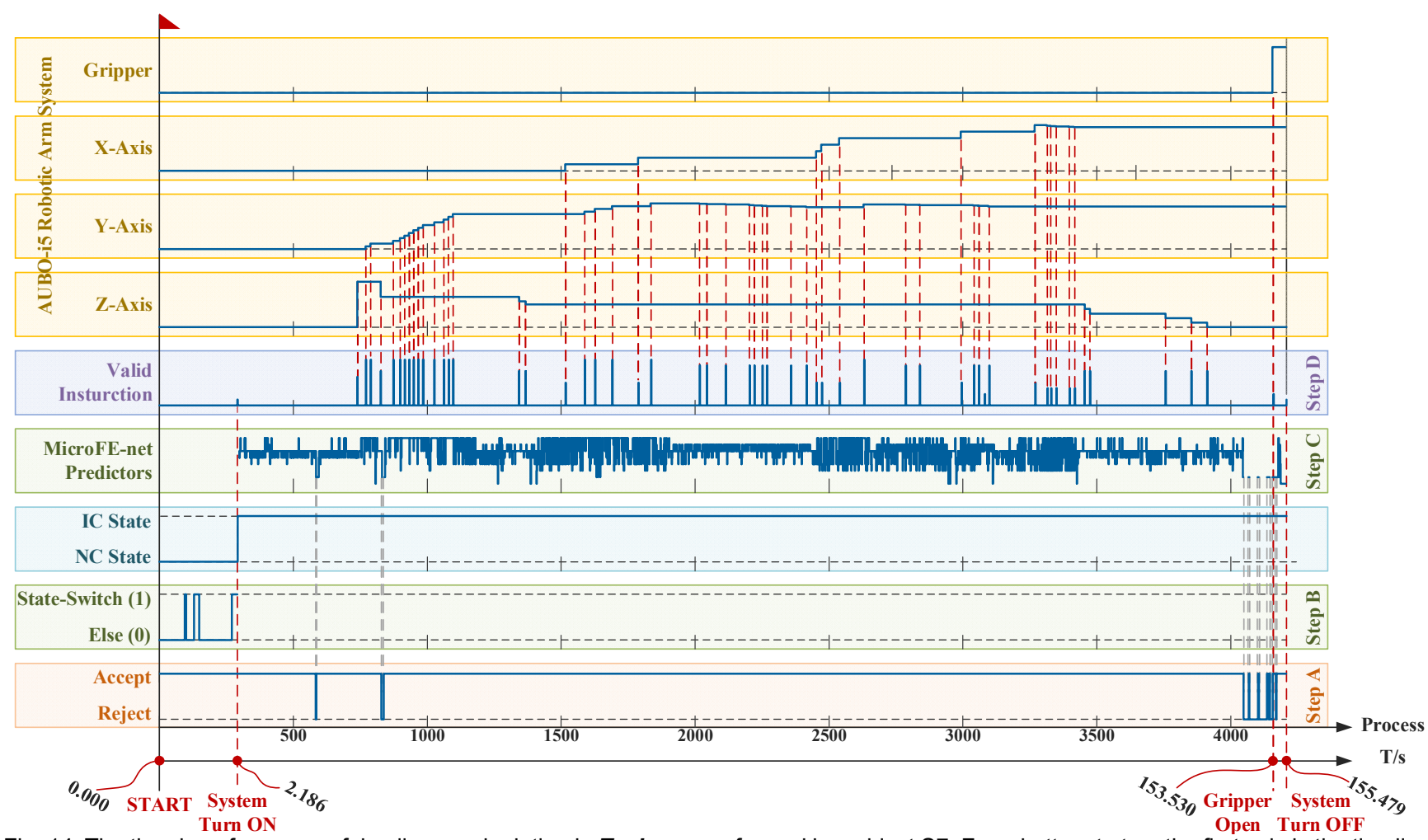

Fig. 14. The time log of a successful online manipulation in Task one performed by subject S7. From bottom to top, the first axis is the timeline, and the second axis is a counter which represents the processing for $n$th $100 \mathrm{~ms}$ EEGs. The five boxes in the lower area illustrate the algorithm results along the timeline step-by-step, and the four boxes in the upper area illustrate the AUBO-i5 robotic arm system movement.

According to Fig. 14, firstly the timestamp 0.000 s begins at the 'Start' button being pressed by S7-self; secondly, at 2.186 s S7 switched on the microFE-BCI successfully; thirdly, after carefully movements, S7 opened the electrical gripper to put down the wooden block at $153.530 \mathrm{~s}$; finally, S7 switched off the microFE-BCI and finished the task at 
155.479 s. During the operation, unrelated EEGs were successfully rejected by microFE-BCI system. The final deviation for the wooden block in Fig. 14's trial is $-4.5 \mathrm{~mm}$ on Xaxis and $+3.1 \mathrm{~mm}$ on Y-axis (Fig. 15). The placement deviation with the microFE-BCI is shown in Fig. 16, along with the deviation by FlexPendant.

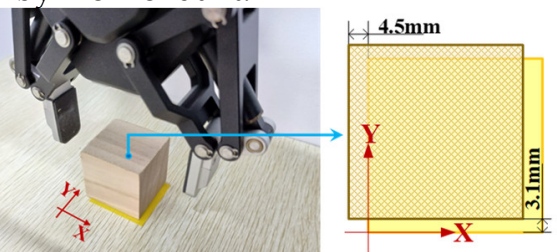

Fig. 15. The illustration for placement deviation in Task one.

For a reliable control method, under one's visual guidance, the deviation shall appear certain random volatility within a small range. Instead of perfect zero deviation, the variation range of devation under microFE-BCI is of more concerned. Among all online subjects (S7-S16), the averaged variation range (AVR) of microFE-BCI's deviation is $11.14 \pm 6.13 \mathrm{~mm}$ along $\mathrm{X}$-axis and $9.78 \pm 3.83 \mathrm{~mm}$ along $\mathrm{Y}$-axis, and $10.46 \pm 5.15 \mathrm{~mm}$ in total (while the AVR of FlexPendant is $7.75 \pm 7.67 \mathrm{~mm}$ in total, and shows no significant difference with $\mathrm{P}=0.2089>0.05)$. As in Fig. 16, for most subjects, compared with FlexPendant, deviation by microFE-BCI achieves a comparable range of variation, a similar averaged value, and a close Std, which indicates an approximative level of controllability as FlexPendant. But for few subjects, on one certain axis (i.e., the X-axis of S11) where the median of microFE-BCI's boxplot is marked far from the average, it indicates that there exists uncontrollability during the position adjustment along that axis by using microFE-BCI. Overall, the maximum deviation with microFE-BCI can largely be limited below $20 \mathrm{~mm}$ (S8), from which the minimum deviation can sometimes be \pm 0.1 $\mathrm{mm}(\mathrm{S} 10, \mathrm{~S} 11)$, and none of the subject' deviations shows significant difference compared between microFE-BCI and FlexPedant. The intersection over union (IoU) between the target location B and the block placement in Task one is demonstrated in Fig. 17.

Among all 50 trials, $96 \%$ of trials succeed, only 2 trials by $\mathrm{S} 8$ failed halfway. The averaged IoU of microFE-BCI is $60.03 \pm 11.53 \%$, while $62.05 \pm 6.01 \%$ of FlexPendant, and shows no significant difference with $\mathrm{P}=0.6521>0.05$. As in Fig. 17, the minimum IoU with microFE-BCI among all is $21.47 \%$ (S8) while the maximum is $82.62 \%$ (S13 \& S15). Compared with FlexPendant, statistical test indicates that the IoU of microFE-BCI shows similar performance overall. But for several subjects (e.g., S8, S9, S11 \& S14), the IoU of FlexPendant performed slightly better and scored higher than microFE-BCI, which indicates that during an actual application process the operation under microFE-BCI is more complicated.

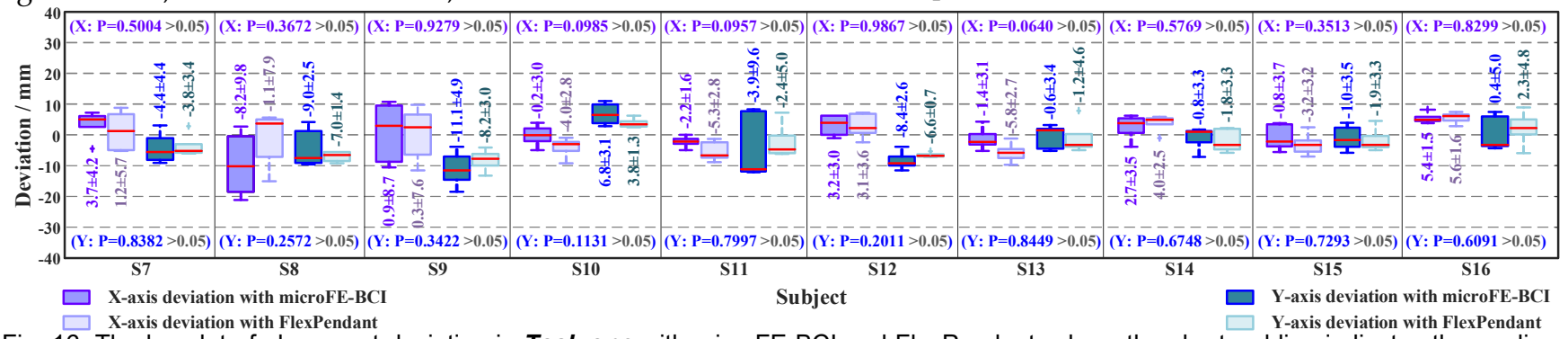

Fig. 16. The boxplot of placement deviation in Task one with microFE-BCl and FlexPendant, where the short red line indicates the median, and the number indicates the mean.

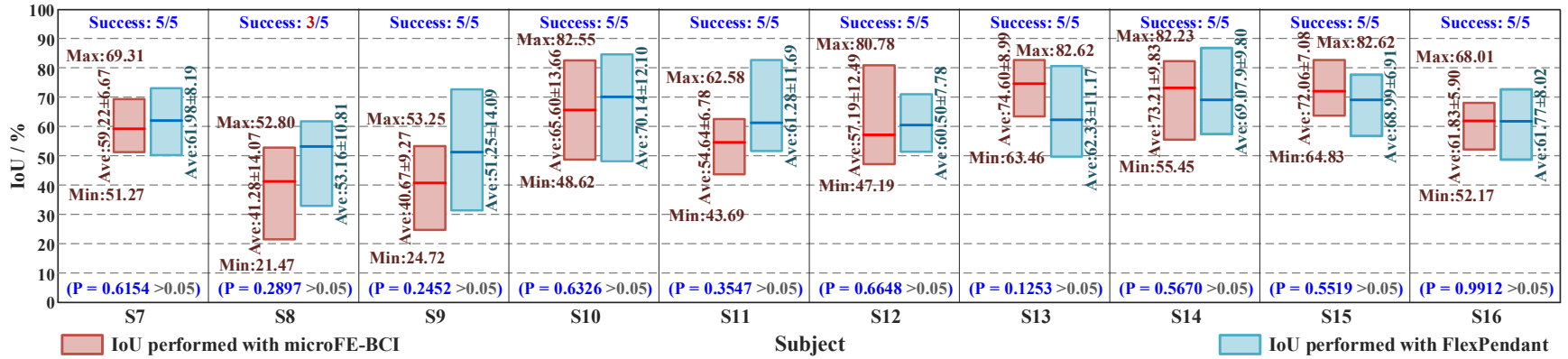

Fig. 17. The loU of placement deviation in Task one with microFE-BCl and FlexPendant, where the short red line indicates the average.

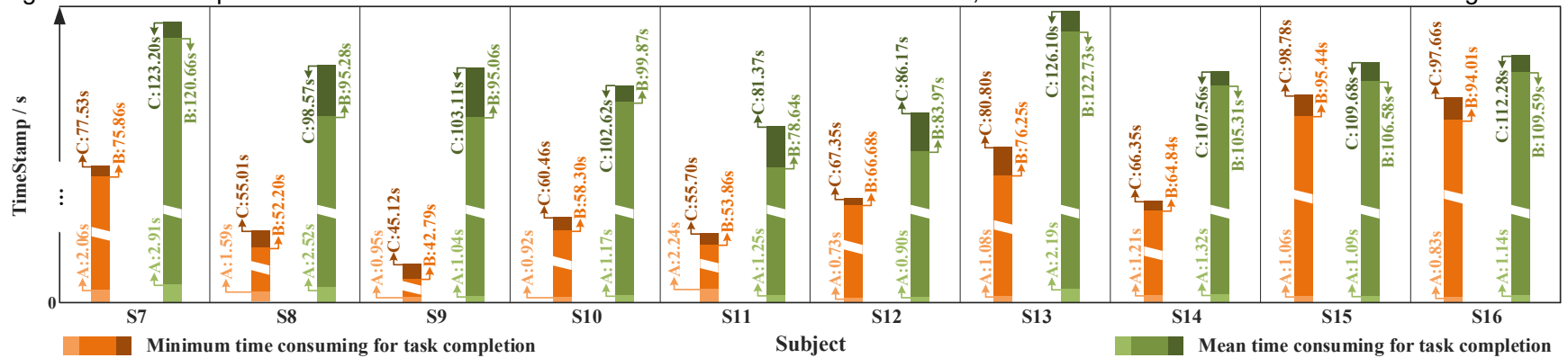

Fig. 18. The time cost in Task one with microFE-BCl, where the timestamp A corresponds to 'System Turn ON', the timestamp B corresponds to 'Gripper Open' and the timestamp C corresponds to 'System Turn OFF' (as demonstrated in Fig. 14).

In terms of time cost (Fig. 18), affecting by different levels of tension and proficiency, the completion time for 
Task one varies from less than $60 \mathrm{~s}$ to approximately $150 \mathrm{~s}$. The average completion time for Task one is $105.07 \pm 13.50 \mathrm{~s}$; in which the average time spent for 'System Turn $\mathrm{ON}^{\prime}$ is $1.55 \pm 0.67 \mathrm{~s}$, for block moving and placing is $100.19 \pm 13.17 \mathrm{~s}$ and for 'System Turn OFF' is $3.27 \pm 1.64 \mathrm{~s}$.

\section{- Task two: Water-pouring with a Prosthetic Hand}

With a 2-DoFs prosthetic hand, only 4 microFEs achieved higher decoding accuracy were enabled. Apart from these 4 microFEs, other valid instructions generated from the rest microFEs would not be transmitted. Slightly differing from Task one, Task two requires a higher real-time response, because a tiny change in wrist angle will pour several milliliters or even dozens of milliliters of water. Table 16 lists the instruction of the 2-DoFs prosthetic hand. Table 17 shows the correspondence between the microFEs and the instruction (left-handed prosthesis), and the consistency checking criteria. Fig. 19 demonstrates the time log of a successful online process in Task two.

$$
\text { TABLE } 16
$$

THE INSTRUCTION LIST OF THE 2-DOFS PROSTHETIC HAND

\begin{tabular}{ccc}
\hline \hline Instruction & System Action & Stepping \\
\hline 'Open' & Palm open & $7.0 \mathrm{~mm}$ \\
'Close' & Palm close & $10.5 \mathrm{~mm}$ \\
'Extorsion' & Wrist external rotation & $11.0^{\circ}$ \\
'Intorsion' & Wrist internal rotation & $12.0^{\circ}$ \\
\hline \hline
\end{tabular}

In Fig. 19, subject switched on the microFE-BCI at 1.329

$\mathrm{s}$, then completed the water-pouring task at $102.367 \mathrm{~s}$, and finally switched off the interface at $106.261 \mathrm{~s}$; The final deviation of water volume in Fig. 19's trial is less than $1 \mathrm{ml}$.

Same as Task one, the deviation affected by the visual perspective and the step distance can hardly be zero even by Joystick. The water volume in Task two is demonstrated in Fig. 20. Among all 50 trials of Task two, 100\% trials successfully finished. The averaged water volume by microFE-BCI is $202.5 \pm 2.5 \mathrm{ml}$, while $202.2 \pm 2.7 \mathrm{ml}$ similarly by Joystick, between which shows no significant difference with $\mathrm{P}=0.7931>0.05$. Compared with Joystick, with such simplified 4 instructions, microFE-BCI achieved almost the same performance as Joystick in water pouring task. The average completion time of Task two is shown in Fig. 21.

The time spent for Task two varies from approximately $60 \mathrm{~s}$ to $150 \mathrm{~s}$. The average completion time for online Task two is $68.41 \pm 19.52 \mathrm{~s}$; Where the averaged time spent for 'System Turn $\mathrm{ON}^{\prime}$ ' is $1.43 \pm 0.45 \mathrm{~s}$, for water pouring is $64.09 \pm 19.06 \mathrm{~s}$ and for 'System Turn OFF' is $2.88 \pm 0.74 \mathrm{~s}$.

$$
\text { TABLE } 17
$$

THe Operational CoRRESPondenCE AND THE ConsistenCy CheCKING CRITERIA $x \%$ FOR SUBJECT S7 IN TASK TWO

\begin{tabular}{lcccccccc}
\hline \hline Item & $\mathrm{m}-$ & $\mathrm{m}-$ & $\mathrm{m}-$ & $\mathrm{m}-$ & $\mathrm{m}-$ & $\mathrm{m}-$ & $\mathrm{m}-$ & $\mathrm{m}-$ \\
& $\mathrm{RB}$ & $\mathrm{FB}$ & $\mathrm{LS}$ & $\mathrm{RS}$ & $\mathrm{OM}$ & $\mathrm{S}$ & $\mathrm{PM}$ & $\mathrm{DM}$ \\
\hline $\begin{array}{l}\text { Instruc- } \\
\text { tion }\end{array}$ & -- & Open & Extorsion & Intorsion & -- & Close & -- & -- \\
Criteria & 0.95 & 0.95 & 0.35 & 0.40 & 1 & 0.95 & 1 & 1 \\
\hline \hline
\end{tabular}

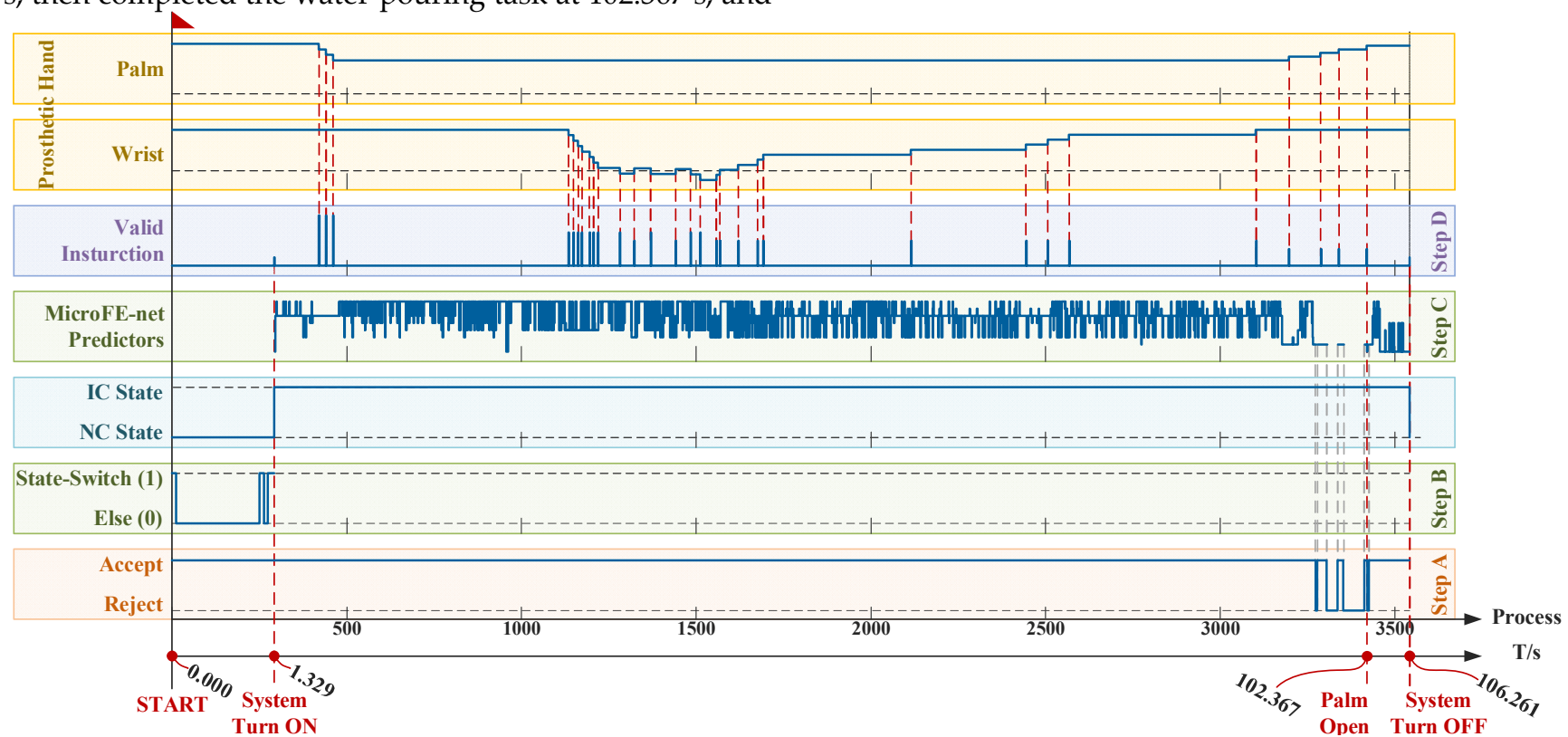

Fig. 19. The time log of a successful online manipulation in Task two. From bottom to top, the first axis is the timeline, and the second axis is a counter which represents the processing for $n$th 100 ms EEGs. The five boxes in the lower area illustrate the algorithm results along timeline step-by-step, and the two boxes in the upper area illustrate the 2-DoFs prosthetic hand movement.

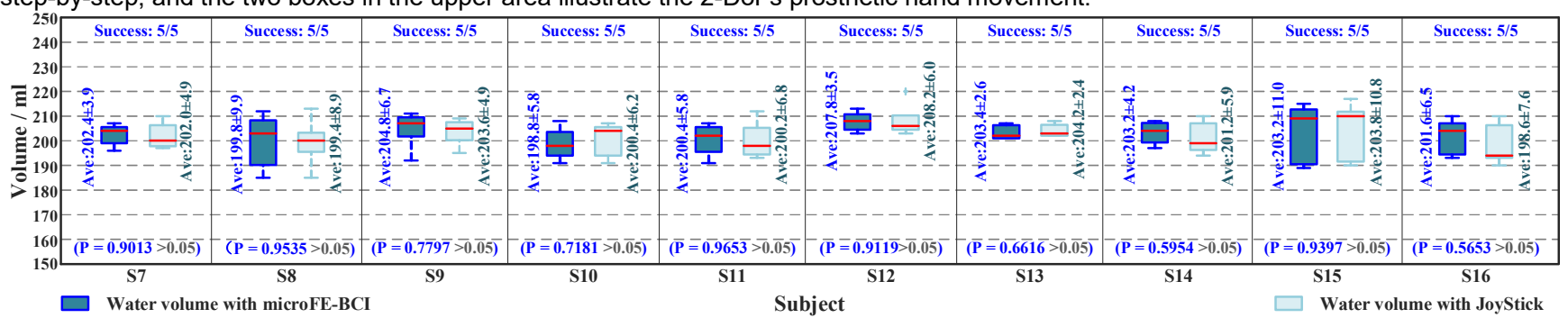

Fig. 20. The boxplot of water volume in online experiment Task two with microFE-BCl and JoyStick, where the short red line indicates the median, and the number indicates the mean. 


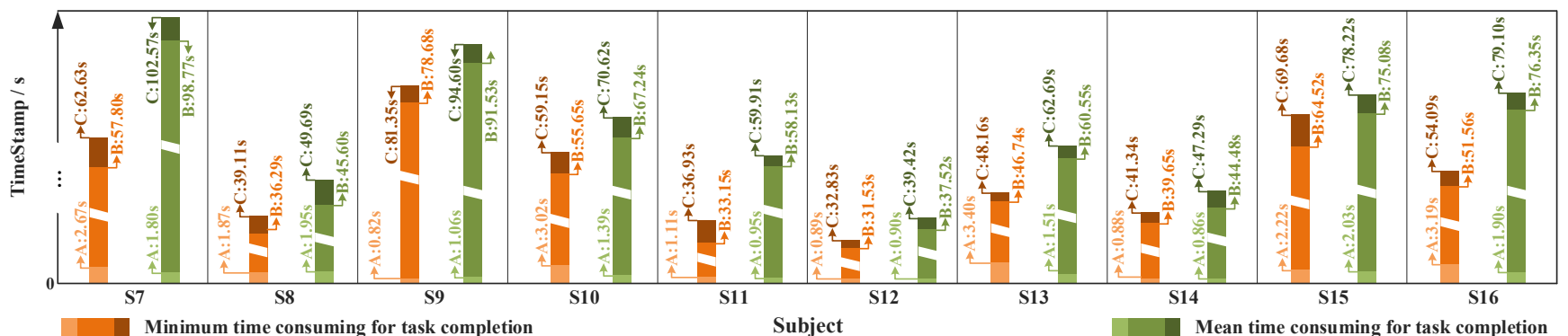

Fig. 21. The time spent in online experiment Task two with the microFE-BCl, where the timestamp A corresponds to 'System Turn ON', the timestamp B corresponds to 'Palm Open' and the timestamp C corresponds to 'System Turn OFF' (as demonstrated in Fig. 19).

\section{Discussion}

To explore the practicality of developed microFE-BCI, more attention was paid to its online application procedure. The overall situation in the online experiment is as follows:

1) During online, subjects need to wear the EEG cap continuously for at least 4 hours, which includes the time for coating conductive gel, training data collection, individual parameters and classifiers computation, consistency checking criteria debugging without peripherals connected, and external device manipulation. Within the rather long wearing time, subjects would drink, eat, and talk for several times with the EEG cap wore.

2) Considering that in the actual operation environment, electromechanical noise is inevitably involved (e.g., the power supply of AUBO-i5), thus during the whole online experiment, the environmental noise is not deliberately controlled. In online process, mobile phones, air conditioners, and machines are all powered on normally, and other laboratory-mates are allowed to enter and exit at will, observe and communicate softly.

3) To give subjects a more realistic experience, after showing an instruction video and giving a brief explanation, the training data collection was conducted by subjects alone, without the experimenter, by operating the software independently.

In online study, there were the following three factors that had been noticed which would affect the performance, and for which our research group planned to conduct a more in-depth study.

\subsection{Long-term Wearing of EEG Cap}

For being tightened by the EEG cap fabric, subjects experienced varying degrees of itching, leading to subconscious scratching, which became more frequent over time. Meanwhile, in the manipulation, under a relatively concentrated state, several subjects sweated beneath the EEG cap. In whole online experiment, subjects might eat (or even have dinner) with the EEG cap worn, and some subjects would move the strap fixed to their chin to facilitate chewing.

Reasons above caused the shift of EEG electrodes which enlarges with the increase of wearing time. Such shift resulted in the difference between the EEG detected in online and collected during acquisition. In terms of impedance, as the wear duration lengthens, the impedance reduced to lower than $1 \mathrm{k} \Omega$, meanwhile, the conductive gel got solidified and dried up. Noticing these, with the proceeding of the online, we have gradually reduced the amount of training data gathering to compress the duration.

\subsection{Mental State}

Being more difficult than commercial control forms (i.e., FlexPendant \& Joystick), subjects were relatively more concentrated in the process of microFE-based control tasks, resulting in varying nervousness depended on personal psychology. In operation, subjects with higher levels of tension were found to show greater degradation from the 'debug' mode (microFE-BCI without peripherals connected) to the actual microFE-BCI based electromechanics control; On the contrary, the performance of tranquil subjects remained consistent. In addition, by lessening the control instructions, Task two shows the decrease of difficulty, the relief of nervousness, and the improvement of proficiency. Thus, even Task two requires more operation agility, the overall performance is more excellent than Task one.

In the whole online study, we have observed that the mental state of subjects has a great impact on the completion quality of control tasks, inspired by which we've also carried out a study on the compensation method of manipulation quality affected by mental state.

\subsection{Physical Movements}

In online procedure, subjects were asked to sit comfortably, but avoid extra-large physical movements. For most subjects, body movements were found that would reduce the stability of microFE-BCI during applications. But for few subjects, such as subjects S11 \& S14 who actively asked to stand up, pace, and softly communicate in Task one, physical movements didn't show much influence on the stability of microFE-BCI. The performance of these trials by subjects S11 \& S14 were kept in the result of this paper. Encouraged by these two subjects, we believe that the proposed microFE-BCI is promising of realistic applicability, thereby research work emphasized on the stability under physical movements of microFE-BCI will be conducted later.

\section{CONCLUSIONS}

In this paper, a novel practical asynchronous real-time EEG-based control paradigm assisted by micro-facial-expressions (microFE-BCI) with 8 valid control instructions was proposed and implemented. It is considered as an upgrade of the FE-BCI, and was applied to various online electromechanical manipulation tasks for evaluating its paracticality, especially stability and agility.

Firstly, with brain connectivity analysis, the high participation of the motor cortex under microFE-BCI was revealed. The directional information interaction conformed 
to the contralateral control fact, and demonstrated the domination of motor cortex. This analysis provided a deeper understanding of the microFE-BCI.

Secondly, an asynchronous real-time processing algorithm was proposed, along with the microFE-Net for decoding. This core algorithm enabled users to switch on/off interface asynchronously, expanded the instruction sets (to 8 instructions), and reduced the misoperation in traditional literal translation BCI. Each decoded label was generated by the latest $100 \mathrm{~ms}$ EEGs to ensure the real-time capability.

In offline, the microFE-BCI achieved accuracies of $96.46 \% \pm 1.07$ for interface switching and $92.68 \% \pm 1.21$ for microFE-BCI-based real-time control. The theoretical timespan microFE-BCI-based instruction was $105.5 \mathrm{~ms}$ in maximum, timing from the generation of microFE-EEGs.

In online, for Task one emphasizing more stability, the microFE-BCI performed overall $96 \%$ success rate with $60.03 \pm 11.53 \%$ averaged IoU, which showed the same level of controllability as FlexPendant $(\mathrm{P}=0.6521>0.05)$. For Task two emphasizing more agility, the microFE-BCI performed $100 \%$ success rate with $202.5 \pm 7.0 \mathrm{ml}$ averaged water volume, which achieved almost the same score as Joystick $(\mathrm{P}=0.7931>0.05)$.

The research suggested the capability and flexibility of the microFE-BCI for manipulation applications, with a similar level of controllability and agility as the commercial control methods. During the study, more subtle factors that may affect the online performance have been noticed by us, on which we will carry out more in-depth research. We sincerely look forward to the realization of 'brain control' in real-world applications in the future.

\section{ACKNOWLEDGMENT}

This work was supported by the National Key Research and Development Program of China under Grant 2017YFB1300303.

\section{REFERENCES}

[1] H. Berger, "Über das Elektrenkephalogramm des Menschen," Archiv für Psychiatrie und Nervenkrankheiten, vol. 87, no. 1, pp. 527570, 1929/12/01, 1929.

[2] J. J. Vidal, "TOWARD DIRECT BRAIN-COMPUTER COMMUNICATION," Annual Review of Biophysics and Bioengineering, vol. 2, pp. 157-180, 1973.

[3] A. J. Casson, D. C. Yates, S. J. M. Smith et al., "Wearable Electroencephalography," IEEE Engineering in Medicine and Biology Magazine, vol. 29, no. 3, pp. 44-56, 2010.

[4] R. Abiri, S. Borhani, E. W. Sellers et al., "A comprehensive review of EEG-based brain-computer interface paradigms," Journal of Neural Engineering, vol. 16, no. 1, pp. 011001, 2019/01/09, 2019.

[5] L. Georgescu, D. Wallace, D. Kyong et al., "The Future of Work: Towards Service Robot Control through Brain-Computer Interface," in 2020 10th Annual Computing and Communication Workshop and Conference (CCWC), 2020, pp. 0932-0937.

[6] H. J. Hwang, S. Kim, S. Choi et al., "EEG-Based Brain-Computer Interfaces: A Thorough Literature Survey," International Journal of Human-Computer Interaction, vol. 29, no. 12, pp. 814-826, Dec, 2013.
[7] D. Zhu, J. Bieger, G. Garcia Molina et al., "A survey of stimulation methods used in SSVEP-based BCIs," Computational intelligence and neuroscience, pp. 702357, 2010 (Epub 2010 Mar, 2010.

[8] G. R. Muller-Putz, and G. Pfurtscheller, "Control of an Electrical Prosthesis With an SSVEP-Based BCI," IEEE Transactions on Biomedical Engineering, vol. 55, no. 1, pp. 361-364, 2008.

[9] J. Han, M. Xu, Y. Wang et al., "'Write' but not 'spell' Chinese characters with a BCI-controlled robot," Annual International Conference of the IEEE Engineering in Medicine and Biology Society. IEEE Engineering in Medicine and Biology Society. Annual International Conference, vol. 2020, pp. 4741-4744, 2020-Jul, 2020.

[10] Z. Lu, X. Zhang, L. Zhang et al., "An ANFIS Method to Improve SSVEP-BCI Anti-blinking Stability," Journal of Vibration, Measurement and Diagnosis, vol. 39, no. 4, pp. 727-732, 2019.

[11] R. Li, X. D. Zhang, H. Z. Li et al., "An approach for braincontrolled prostheses based on Scene Graph Steady-State Visual Evoked Potentials," Brain Research, vol. 1692, pp. 142-153, Aug, 2018.

[12] L. M. Zhang, X. D. Zhang, Z. F. Lu et al., "A New Object-oriented SSVEP-based BCI Paradigm Using Continuous Action Scene," 2017 Ieee 7th Annual International Conference on Cyber Technology in Automation, Control, and Intelligent Systems, IEEE Annual International Conference on Cyber Technology in Automation Control and Intelligent Systems, pp. 1078-1082, New York: Ieee, 2017.

[13] X. G. Chen, Y. J. Wang, M. Nakanishi et al., "High-speed spelling with a noninvasive brain-computer interface," Proceedings of the National Academy of Sciences of the United States of America, vol. 112, no. 44, pp. E6058-E6067, Nov, 2015.

[14] J. R. Wolpaw, N. Birbaumer, D. J. McFarland et al., "Braincomputer interfaces for communication and control," Clinical Neurophysiology, vol. 113, no. 6, pp. 767-791, Jun, 2002.

[15] N. Sharma, V. M. Pomeroy, and J. C. Baron, "Motor imagery - A backdoor to the motor system after stroke?," Stroke, vol. 37, no. 7, pp. 1941-1952, Jul, 2006.

[16] G. Pfurtscheller, C. Guger, G. Muller et al., "Brain oscillations control hand orthosis in a tetraplegic," Neuroscience Letters, vol. 292, no. 3, pp. 211-214, Oct 13, 2000.

[17] B. J. Edelman, B. Baxter, and B. He, "EEG Source Imaging Enhances the Decoding of Complex Right-Hand Motor Imagery Tasks," Ieee Transactions on Biomedical Engineering, vol. 63, no. 1, pp. 4-14, Jan, 2016.

[18] G. Pfurtscheller, B. Z. Allison, C. Brunner et al., "The hybrid BCI," Frontiers in neuroscience, vol. 4, pp. 30, 2010, 2010.

[19] G. Pfurtscheller, T. Solis-Escalante, R. Ortner et al., "Self-Paced Operation of an SSVEP-Based Orthosis With and Without an Imagery-Based "Brain Switch:" A Feasibility Study Towards a Hybrid BCI," Ieee Transactions on Neural Systems and Rehabilitation Engineering, vol. 18, no. 4, pp. 409-414, Aug, 2010.

[20] Y. Q. Li, J. H. Pan, F. Wang et al., "A Hybrid BCI System Combining P300 and SSVEP and Its Application to Wheelchair Control," Ieee Transactions on Biomedical Engineering, vol. 60, no. 11, pp. 3156-3166, Nov, 2013.

[21] Z. F. Lu, X. D. Zhang, H. Z. Li et al., "A Real-Time Brain Control Method Based on Facial Expression for Prosthesis Operation," in 2018 Ieee International Conference on Robotics and Biomimetics, New York, 2018, pp. 668-673.

[22] X. Zhang, J. Guo, R. Li et al., "A Simulation Model and Pattern Recognition Method of Electroencephalogram Driven by Expression," Journal of Xi'an Jiaotong University, vol. 50, no. 6, pp. 
1-8, 2016, 2016.

[23] R. Li, X. D. Zhang, Z. F. Lu et al., "An Approach for BrainControlled Prostheses Based on a Facial Expression Paradigm," Frontiers in Neuroscience, vol. 12, pp. 15, Dec, 2018.

[24] Z. Lu, X. Zhang, R. Li et al., "A Brain Control Method for Prosthesises Based on Facial Expression," China Mechanical Engineering, vol. 29, no. 12, pp. 1454-1459,1474, 2018, 2018.

[25] Z. Lu, X. Zhang, H. Li et al., "A Semi-Asynchronous Real-Time Facial Expression Assisted Brain Control Method: An Extension" in 2020 The 10th IEEE International Conference on CYBER Technology in Automation, Control, and Intelligent Systems, Xi'an, China, 2020.

[26] D. Z. Yao, "A method to standardize a reference of scalp EEG recordings to a point at infinity," Physiological Measurement, vol. 22, no. 4, pp. 693-711, Nov, 2001.

[27] W. M. Association, "World Medical Association Declaration of Helsinki: Ethical Principles for Medical Research Involving Human Subjects," JAMA, vol. 310, no. 20, pp. 2191-2194, 2013.

[28] V. Sakkalis, "Review of advanced techniques for the estimation of brain connectivity measured with EEG/MEG," Computers in Biology and Medicine, vol. 41, no. 12, pp. 1110-1117, Dec, 2011.

[29] K. J. Friston, "Functional and effective connectivity: a review," Brain connectivity, vol. 1, no. 1, pp. 13-36, 2011, 2011.

[30] B. Horwitz, "The elusive concept of brain connectivity," Neuroimage, vol. 19, no. 2, pp. 466-470, Jun, 2003.

[31] L. Sommerlade, K. Henschel, J. Wohlmuth et al., "Time-variant estimation of directed influences during Parkinsonian tremor," Journal of Physiology-Paris, vol. 103, no. 6, pp. 348-352, Nov, 2009.

[32] G. Nolte, O. Bai, L. Wheaton et al., "Identifying true brain interaction from EEG data using the imaginary part of coherency," Clinical Neurophysiology, vol. 115, no. 10, pp. 22922307, Oct, 2004.

[33] G. Gomezherrero, "Brain Connectivity Analysis with EEG," Tampere University of Technology, 2010.

[34] C. W. J. Granger, "INVESTIGATING CAUSAL RELATIONS BY ECONOMETRIC MODELS AND CROSS-SPECTRAL METHODS," Econometrica, vol. 37, no. 3, pp. 424-438, 1969.

[35] J. Geweke, "MEASUREMENT OF LINEAR-DEPENDENCE AND FEEDBACK BETWEEN MULTIPLE TIME-SERIES," Journal of the American Statistical Association, vol. 77, no. 378, pp. 304-313, 1982.

[36] L. A. Baccala, and K. Sameshima, "Partial directed coherence: a new concept in neural structure determination," Biological Cybernetics, vol. 84, no. 6, pp. 463-474, Jun, 2001.

[37] A. H. Omidvarnia, M. Mesbah, M. S. Khlif et al., "Kalman filterbased time-varying cortical connectivity analysis of newborn EEG," in 2011 Annual International Conference of the Ieee Engineering in Medicine and Biology Society, New York, 2011, pp. 1423-1426.

[38] A. Omidvarnia, G. Azemi, B. Boashash et al., "Measuring TimeVarying Information Flow in Scalp EEG Signals: Orthogonalized Partial Directed Coherence," Ieee Transactions on Biomedical Engineering, vol. 61, no. 3, pp. 680-693, Mar, 2014.

[39] H. Hytti, R. Takalo, and H. Ihalainen, "Tutorial on multivariate autoregressive modelling," Journal of clinical monitoring and computing, vol. 20, no. 2, pp. 101-8, 2006 Apr (Epub 2006 May, 2006.

[40] L. Faes, and G. Nollo, "Extended causal modeling to assess Partial Directed Coherence in multiple time series with significant instantaneous interactions," Biological Cybernetics, vol.
103, no. 5, pp. 387-400, Nov, 2010.

[41] H. Akaike, "Information theory and an extension of the maximum likelihood principle," 2nd international symposium on information theory. Abstracts of papers, pp. 276-276, 1971, 1971.

[42] A. H. Omidvarnia, G. Azemi, B. Boashash et al., "Orthogonalized Partial Directed Coherence for Functional Connectivity Analysis of Newborn EEG," Neural Information Processing, Iconip 2012, Pt Ii, Lecture Notes in Computer Science T. Huang, Z. Zeng, C. Li et al., eds., pp. 683-691, Berlin: Springer-Verlag Berlin, 2012.

[43] M. Zavaglia, L. Astolfi, F. Babiloni et al., "A neural mass model for the simulation of cortical activity estimated from high resolution EEG during cognitive or motor tasks," Journal of Neuroscience Methods, vol. 157, no. 2, pp. 317-329, Oct, 2006.

[44] R. T. Lauer, P. H. Peckham, K. L. Kilgore et al., "Applications of cortical signals to neuroprosthetic control: A critical review," Ieee Transactions on Rehabilitation Engineering, vol. 8, no. 2, pp. 205-208, Jun, 2000.

[45] L. Derczynski, Complementarity, F-score, and NLP Evaluation, Paris: European Language Resources Assoc-Elra, 2016.

[46] Y. Bengio, and Y. Lecun, "Convolutional Networks for Images, Speech, and Time-Series," 11/10, 1997.

[47] D. E. Rumelhart, G. E. Hinton, and R. J. Williams, “Learning representations by back-propagating errors," Nature, vol. 323, pp. 533, October 01, 1986, 1986.

[48] F. A. Gers, J. Schmidhuber, and F. Cummins, "Learning to forget: continual prediction with LSTM." pp. 850-855 vol.2.

[49] S. Hochreiter, and J. Schmidhuber, "Long Short-Term Memory," vol. 9, no. 8, pp. 1735-1780, 1997.

[50] N. Srivastava, G. Hinton, A. Krizhevsky et al., "Dropout: A Simple Way to Prevent Neural Networks from Overfitting," Journal of Machine Learning Research, vol. 15, pp. 1929-1958, Jun, 2014.

[51] K. Simonyan, and A. Zisserman, "Very Deep Convolutional Networks for Large-Scale Image Recognition," arXiv 1409.1556, 09/04, 2014.

[52] G. Huang, Z. Liu, L. van der Maaten et al., Densely Connected Convolutional Networks, 2017.

[53] K. He, X. Zhang, S. Ren et al., Deep Residual Learning for Image Recognition, 2016.

[54] K. K. Ang, Z. Y. Chin, H. H. Zhang et al., "Filter Bank Common Spatial Pattern (FBCSP) in Brain-Computer Interface," 2008 Ieee International Joint Conference on Neural Networks, Vols 1-8, IEEE International Joint Conference on Neural Networks (IJCNN), pp. 2390-2397, New York: Ieee, 2008.

Xiaodong Zhang received the B.S. degree in thermal energy and power engineering, M.S. degree, and Ph.D. degree in mechanical engineering from Xi'an Jiaotong University, Xi'an, China, in 1982, 1992, and 1996, respectively. He is currently a professor and Ph.D. Supervision with the College of Mechanical Engineering, Xi'an Jiaotong University. More than 130 papers have been published in his research career. His main research interests include intelligent robotics, intelligent measurement, diagnosis \& control technology, and Bio-signals measurement technology and its applications in engineering. 